Körper des 7wöchentlichen Kindes. Auch an diesem stellte sich mit der localisirten Beugung der Brustgegend hei geradestehender Lenden- und Halswirbelsäule sogleich Lordose und spiralige Drehung ein. Was aber diesen Versuch vor dem früher erwähnten auszeichnet, ist der Umstand, dass an dem betreffenden Körper das Brustbein mil den Rippenknorpeln beseitigt war, und dass dennoch die bezeichnende Gestalt des Thorax sich einstellte. Es war hierbei noch besonders zu bemerken, dass der freie Rand beider Brusthälften in diesem Versuche eine Abweichung von seiner Lage nicht erkennen liess, und dass die gegenseitige Entfernung beider Ränder, von der Schnittfläche des fünften Rippenknorpels der einen Seite zu dem entsprechenden Punkte der anderen Seite gemessen, durch den Versuch nicht verändert wurde. - Durch diese Erfahrung wird die oben bereits angegebene Selbständigkeit jeder Brustwand in der skoliotischen Veränderung auch noch unmittelbarer auf experimentałem Wege nachgewiesen.

\title{
XVI.
}

\section{Beitrag zur Lehre von der pathologischen örtlichen und allgemeinen Wärmebildung.}

Von Dr. Schröder, Assistenzarzt an der geburtshülflich - gynäkologischen klinik in Bonn.

Von der Ueberzeugung ausgehend, dass die Theilung der Arbeit, wie sie heute auch auf dem Gebiete der medicinischen Thätigkeit besteht und wie sie bei den riesenbaften Fortschritten der einzelnen medicinischen Disciplinen und ihrer Hülfswissenschaften nothwendig geworden ist, nur dann den Gesammtfortschritt und die Weiterbildung der theoretischen Anschauungen, die ja immer wieder anregend und neue Bahnen brechend auf die Praxis einwirken, fördern kann, wenn der Specialist, statt in handwerksmässige Weiterbildung, seiner Branche und in schablonenmässige $\mathrm{Be}-$ handlung der einzelnen $\mathrm{Zweige} \mathrm{zu}$ verfallen, stets als leitenden Faden die Fortscbritte der Physiologie und allgemeinen Pathologie 
vor Augen behält und dass er, wenn er diese Fortschritte für sein Specialfach verwendet, andererseits auch die Pflicht hat, in geeigneten Fällen durch Beobachtung am klinischen Material das seinige zur Lösung brennender theoretischer Tagesfragen beizutragen von dieser Ueberzeugung ausgehend, halte ich, obgleich durch meine BeschäPtigung eigentlich physiologischen Fragen ferner stehend, es nicht für unmotivirt, folgende kleine Abhandlung über örtliche und allgemeine Wärmebildung dem Urtheil des Pathologen zu unterbreiten.

Sind auch die aus meinen Beobachtungen gezogenen Folgerungen nicht ganz neu, so glaube ich doch, dass zur Lösung einer so wichtigen Frage, wie die über das Verbältniss der örtlichen pathologischen Wärmeproduction zur allgemeinen fieberhaften Temperatursteigerung ein Jeder, der Gelegenheit hat, derartige Beobachtungen zu machen, das seinige beitragen muss. Denn dass diess Verhältniss nicht ein ganz einfaches und leicht zu ergründendes ist, geht schon daraus hervor, dass Forscher, wie 0 . Weber und Billroth nicht durch theoretische Schlüsse, sondern auf dem Wege der experimentellen Beobachtung zu direct entgegengesetzien Resultaten über die Erzeugung örtlicher Wärme bei Entzündungen gelangen konnten. Denn während Weber übereinstimmend mit älteren Untersuchern in den meisten Fällen eine örtliche Temperatursteigerung nachweisen konnte, gelang diess Billroth unter 41 Fällen nur zweimal. Ein Hauptgrund dieser abweichenden Resultate liegt jedenfalls in der Kleinbeit der Zablen, die hier schon a priori zu erwarten sind. Denn wenn auch in einem Krankheitsheerd eine die normale Körperwärme bedeutend übersteigende Temperatur producirt wird, so muss dieselbe jedenfalls bei der ungeheuren Schnelligkeit des Blutkreislaufes, der beim Menschen in weniger als einer halben Minute vollendet ist, so schnell durch den ganzen Körper gleichmässig verbreitet werden, dass man in dem Heerd selbst nur einen geringen Ueberschuss wird erwarten können, ja dass bedeutende Unterschiede, wie Weber sebr richtig bemerkt, wenn sie sich finden sollten, mit Misstrauen betrachtet werden müssten. Die Kleinheit der Zablen bedingt es schon an und für sich, dass eine Beobachtung nicht beweisend ist, sondern dass nur eine grössere Anzahl von Beobachtungen, die auf statistische Weise behandelt, ein wenn auch kleines doch entschiedenes 
Resultat geben, einzig und allein beweisen können. Wenn in Anbetracht dieser Thatsache mein beobachtetes Material zur statistischen Behandlung immerhin klein erscheinen möchte, so muss ich zu meiner Entschuldigung die Schwierigkeit dieser Beobachtungen und den bedeutenden Zeitaufwand, den sie erfordern, wenn sie wirklich gewissenhaft angestellt werden, anführen. Dass übrigens meine Resultate unter sich vollständig übereinstimmend ausgefallen sind, glaube ich hauptsächlich dem geeigneten Material zu verdanken. Denn wenn die bisherigen Beobachter sich nicht immer mit Erfolg bemühten, eine die Körperwärme übersteigende Temperatur in offnen Wunden nacbzuweisen, so lag die Schuld daran jedenfalls in den der Abküblung so günstigen Verhältnissen, denen die offne Wunde im Vergleich zu dem hermetisch schliessenden After oder der gleichfalls gut schliessenden Achselhöhle ausgesetzt ist, und Weber' hat insofern Recht, wenn er zwei Versuchen Billroth's, die ein positives Resultat ergaben, eine höhere Beweiskraft zuspricht, als den 39 andern. Ein weit günstigeres Feld der Beobachtung als offene Wunden sind die weiblichen Genitalien, die durch ihre Lage vor Abkühlung geschützt, die Einführung des Thermometers sehr bequem gestatten, und eine besonders geeignete Zeit ist die Zeit während und nach der Geburt, in der die Genitalien den verschiedensten pathologischen Zuständen ausgesetzt sind und in der sich geeignete Thermometer mit leichter Mühe bis in den Uterus einbringen lassen.

Bevor ich die Resultate meiner Beobachtungen mittheile, mag es mir vergönnt sein, in kurzen Worten die Methode meiner Untersuchung darzulegen. Die zu den Messungen benutzten Instrumente waren zum Theil Leyser'sche in funftel Grade getheilte Thermometer, an denen sich Zehntelgrade mit vollständiger Sicherheit und Zwanzigstel annähernd sicher abschätzen liessen. Unter acht Leyser'schen Thermometern waren vier vollständig gleich; die übrigen wurden zu diesen Messungen nur ausnahmsweise benutzt und dann natürlich die Abweichungen genau regulirt. Ausserdem wurden zu den feineren Beobachtungen zwei in zwanzigstel Grade getheilte sehr genau gearbeitete Thermometer von Geissler gebraucht, von denen eins zum Zweck seiner leichteren Einführung in den Uterus am unteren Ende nach Art einer Uterussonde gekrümmt war. Sämmtliche benutzte Thermometer wurden von Zeit 
zu Zeit, meistens alle 8 Tage, genau verglichen und etwaige Schwankungen durch Rechnung regulirt. Es ist das häufige Vergleichen bei derartigen Messungen durchaus nothwendig, da besonders neue Thermometer, wenn sie auch noch so genan gearbeitet sind, leicht ihren Eispunkt verändern. Die Messungen selbst wurden ohne Ausnabme von mir persönlich in der Weise angestellt, dass die Thermometer genau - mit besonderer Aufmerksamkeit stets der in der Achselhöhle gelegenen als am leichtesten zu Fehlerquellen geneigten - gelegt wurden und dass sie dann, nachdem die in der Achsel wenigstens 20 Minuten, die übrigen mindestens 10 Minuten gelegen hatten, eine längere Zeit hindurch in bestimmten Zeitabschnitten - z. B. von $10 \mathrm{zu} 10$ Minuten - abgelesen und der Stand des Quecksilbers notirt wurde. Je nach den übereinstimmenden Resultaten wurde diess längere Zeit - bis zu 3 und 4 Stunden in einzelnen Fällen - fortgesetzt. Es ist das bei Messungen, die eine solche Genauigkeit wie die unten angeführten erfordern, durchaus nothwendig und ist das Verlangen, die Thermometer liegen $\mathrm{zu}$ lassen, bis sie 5 Minuten hindurch dieselbe Temperatur zeigen bei genauen Instrumenten in vielen Fällen der vorhandenen Tagesschwankungen wegen nicht zu erfüllen.

Aus diesen einzelnen Aufzeichnungen, deren Anzahl von 3-16 wechselt - so z. B. bestehen die 539 in Rechnung gezogenen Messungen an Wöchnerinnen aus 2932 einzeln notirten Beobachtungen - erschien es mir sicherer, die geeignet erscheinende ratjonell auszuwählen, als durch Rechnung den Durchschnittswerth zu finden. Denn einzelne charakterisirten sich durch ibr Verhältniss zu den übrigen sofort als durch Fehlerquellen - nicht genaues Anschliessen des Arms u. dergl. - bedingt und hätten also in die Rechnung leicht zu vermeidende Fehler gebracht.

Zuerst nun erschien es wichtig, um eỉnen Maassstab für Abweichungen von der Regel zu hahen, das Verbältniss zwischen den Temperaturen an den einzelnen benutzten Körpergegenden unter normalen Verhältnissen festzustellen. Am unconstantesten fand ich das Verhältniss zwischen der Temperatur der Achselhöhle und der des Rectum. Unter den vorhandenen Angaben sind die von Fick*), die nach 3 Versuchspersonen im Durchschnitt berechnet und auf 
Celsius reducirt*), für die einzelne Messung einen Unterschied von über $3^{0}$ geben, viel zu hoch. Bärensprung**) fand bei Kindern einen Unterschied von $0,4^{\circ}$, während Ziemssen und Krablir ${ }^{* * *}$ ) bei Erwachsenen im Mittel $0,2^{0}$ fanden. Wie verschieden aber auch ihre Resultate ausfielen, zeigt die Maximalangabe von $0,55^{\circ}$, der Minimalangabe von $0,0^{\circ}$ gegenüber. Drei an einem gesunden kräftigen jungen Manne von mir angestellte Messungen ergaben $0,6^{\circ}, 0,47^{\circ}$ u. $0,46^{\circ}$ Unterschied, so dass sich danach die mittlere Differenz auf $0,51^{\circ}$ stellen würde. 35 von mir an 17 Schwangeren angestellte Messungen ergaben im Mittel $0,156^{\circ}$ (im Maximum $0,6^{\circ}$, im Minimum $0,0^{\circ}$ ). Man ersieht aus diesen Angaben, wie sehr schwankend das Verhältniss ist. Da ausserdem das Einführen des Thermometers in das Rectum den betreffenden Personen stets sehr unangenehm war, während sie die Einführung in die Vagina sich leicht gefallen liessen, so sah ich von weiteren Messungen im Rectum $a b$ und bemühte mich, den normalen Unterschied zwischen den Temperaturen der Achsel und der Vagina festzustellen.

In der Literatur finden sich darüber nur sehr spärliche Angaben. Bärensprung $\dagger$ ) bemerkt, dass die Schwangerschaft eben so wenig wie die Menstruation die Temperatur der Frauen verändere. Doch scheint es nach seinen Beobachtungen, als ob, wenn überhaupt ein Unterschied stattindet, die Scheidentemperatur bei Schwangern etwas erhöht ist. Das Mittel aus 6 Messungen bei Nichtschwangern beträgt nämlich $37,7^{\circ}$, aus 5 Messungen bei Schwangern $37,725^{\circ}$. Da ich über diess Verbältniss keine weiteren Angaben finden konnte und mir selbst ein Material von Scheiden nicht schwangerer, gesunder Personen - denn auch das gynäkologische Material war aus einleuchtenden Gründen hierzu unbrauchbar - nicht zu Gebote stand, glaubte ich dem wahren Verbältniss ziemlich nahe zu kommen, jedenfalls aber die Differenz zwischen Achsel und Scheide nicht zu unterschätzen, worauf

*) Es sind überhaupt, wo im Folgenden nach Réaumur angestellte Messungen angefübrt sind, dieselben der leichteren Vergleichung halber auf Grade der 100theiligen Scala berechnet.

**) Müller's Archiv 1851. S. 148.

***) Greifswalder medic. Beiträge 1863. S. 124 .

t) a. a. 0. S. 157 .

Archiv f. pathol. Anat. Bd, XXXV. Hft. 2. 
es mir, um der Beweiskraft meiner Beobachtungen keinen Eintrag zu thun, hauptsächlich ankam, wenn ich einige Messungen an Schwangern dabei zu Grunde legte. Ich konnte wenigstens kein Moment herausfinden, welches, wenn man die Beine der Versuchsperson genau zusammenlegen lässt, die Scheidentemperatur bei Schwangern der Gesunder gegenüber erniedrigen könnte, während der nahe Uterus - dessen Temperatur in der Schwangerschaft, wie wir weiter unten sehen werden, erhöht ist - und die nicht seltenen entzündlichen Prozesse der Scheide bei Schwangern eine etwas höbere Temperatur voraussetzen lassen. Es wurde nun unter 55 an 19 Schwangern angestellten Messungen 40 Mal die Scheide wärmer als die Achselböhle gefunden, im Maximum um 0,3, im Minimum um 0,05, 12 Mal war die Temperatur gleich und 3 Mal war sie in der Achsel höher und zwar um 0,1 1 Mal und um $0,052 \mathrm{Mal}$. Das mittlere Uebergewicht der Scheidentemperatur beträgt im Durchschnitt 0,101. Diese Zabl stimmt ziemlich genau mit dem Durchschnitt, den man sich aus den von Winkel*) angegebenen correspondirenden Messungen berechnen kann. Derselbe beträgt nämlich aus 6 Messungen berechnet 0,108 , wäbrend die Schwankungen bei Winkel geringer sind (Maximum 0,15, Minimum 0,075), was sich aus der geringeren Anzahl der Messungen leicht erklärt. Zwischen Primiparis und Multiparis konnte kein durchgreifender Unterschied gefunden werden. $\mathrm{Ob}-$ gleich also auch die Temperatur der Vagina und die der Achselhöhle eine schwankende Differenz ergibt, die bei demselben Individuum in einem Fall bis zu 0,2 geht, in 2 Fällen 0,15 beträgt, aber sonst 0,1 nicht übersteigt, so war diess Verbältniss doch das bei weitem constanteste, denn sowohl das zwischen Achsel und Rectum, als auch das zwischen Vagina und Rectum war bedeutend grösseren Schwankungen ausgesetzt. Die Differenz zwischen letzteren ging beispielsweise bei der obigen Person bis zu $0,3^{\circ}$.

Wenn man so die normale Scheidentemperatur wohl ohne bedeutende Fehlerquelle auch an Schwangern bestimmen kann, so macht die Bestimmung der normalen Uterustemperatur bedeutend grössere Schwierigkeiten. Einen verhältnissmässig grossen Unterschied fand schon Bärensprung *) zwischen der Temperatur des

*) Monatsschrift für Geburtskunde 1862, Bd. 20. S. 413.

**) a. a. 0. S. 134 . 
schwangeren Uterus und der sonstigen Körpertemperatur. Aus seinen Versuchen an Kanincben geht hervor, dass im Mittel bei nicht trächtigen Thieren die Bauchhöhle um $0,3^{\circ}$ wärmer war als der Uterus und dieser letztere wieder um 0,053 wärmer als die Beckenhöhle, während bei trächtigen Thieren die Wärme des Uterus die der Beckenhöhle um 0,113 und die der Bauchhöhle um 0,363 übertraf, so dass während die Differenz in der Wärme der Bauchböhlen 0,337 betrug, der Unterschied zwischen den Beckenhöhlen 0,95 und der zwischen den Uteris $1^{\circ}$ ausmachte. Bärensprung kommt zu dem Schluss, dass diese höhere Wärme des Uterus von der vom Fötus producirten Eigenwärme herstammt und bestätigt diesen Schluss durch die an bebrüteten Eiern gewonnenen Resultate, sowie mit Wahrscheinlichkeit durch directe Messungen an neugebornen Kindern und ibren Müttern. Ich habe nun bei Schwangern im letzten Monat ein nach Art einer Uterussonde gekrümmtes Thermometer in die Uterushöhle eingeführt und auf diese Weise über die Wärme des schwangeren Uterus folgende Resultate erhalten*). Die Temperatur des Uterus (U) war nach 17 an 7 Schwangern angestellten Messungen im Durchschnitt 0,29 (Maximum 0,5, Minimum 0,1) höher als die der Achselhöhle (A) und 0,156 (Maximum 0,32, Minimum 0,05) höher als die der Vagina (V). Zur Vergleichung einen normalen nicht schwangern menschlichen Uterus zu messen, gelang natürlich nicht; nur in einem Fall von Metrorrhagie und Uterusinfarct gelang es, die Quecksilberkugel in das Cavum uteri einzufuhren und war die Differenz zwischen $A$ und $U 0,25$, zwischen $A$ und $V 0,15$ und zwischen

*) Ich bemerke bierzu, dass ich das Thermometer stets vollständig in das Cavum uteri über den inneren Muttermund hinaus mindestens 2 Zoll zwischen Uteruswand und Frucbtblase eingeführt und immer über eine balbe Stunde habe liegen lassen, ohne dass ich ein einziges Mal den Eintritt von Wehen danach beobachtet hätte. Ich habe diese Messungen begonnen an Personen, die das Ende ihrer Schwangerschaft erreicht hatten und die über leichte Kreuzschmerzen klagten, bej denen also der unmittelbare Eintritt der Geburt 2u erwarten war and habe dabei einigemale gesehen, dass die Schmerzen sich wieder verloren und die Geburt erst nach mehreren Tagen eintrat. Hierdurch ermuthigt, habe ich in geeigneten Fallen, auch bei noch nicht ganz beendigter Schwangerschaft, das Thermometer in den Uterus eingeführt, ohne wie gesagt, auch nur einmal den Eintritt von Wehen danach beobachtet zu haben. 
$\mathrm{U}$ und $\mathrm{V}$ also 0,1. Dieser Fall kann jedoch für normale Verhältnisse natürlich nichts beweisen und muss man, wenn man die normale Wärme eines nicht schwangern Uterus festsetzen will, neben den Bärensprung'schen Versuchen an Kanincben, nach denen derselbe beträchtlich kühler ist als die Bauchhöhle, sich auf aprioristische Raisonnements beschränken. Es kann gewiss nicht zweifelhaft sein, dass ein Organ, welches nicht functionirt und in dem der Stoffwechsel jedenfalls sehr langsam vor sich gebt, nicht höher temperirt ist als die übrigen inneren Organe und somit nicht höher als das Scheidengewölbe. Denn dass die Vagina tief genug gelegen ist, um wirklich die Temperatur der inneren Organe anzuzeigen, geht daraus hervor, dass der Quecksilberstand derselbe bleibt, ob man das Thermometer 2 " oder $6^{\prime \prime}$ tief einführt. Wenn sonach der schwangere Uterus thatsächlich eine höhere Wärme zeigt, so muss diess in besonderen Verbältnissen liegen und ich muss nach meinen Beobachtungen der Ansicht Bärensprung's, dass das Plus der Wärme vom Fötus erzeugt wird, vollständig beistimmen. Ich habe zu dem Zweck an drei neugebornen Kindern Versuche angestellt, babe ebenso wie Bärensprung das Kind sofort nach der Geburt in ein warmes Tuch wickeln lassen und ein Thermometer in den After eingefuhrt. Das Thermometer hatte in einem Fall schon nach 2 Minuten, in zwei Fällen nach 3 Minuten seinen höchsten Stand erreicht und begann dann schnell zu fallen. Bei dem Kind Eistrup, einem 1monatlichen Kinde, betrug die Temperatur des Rectum 2 Minuten nach Einlegung des Thermometer $37,88^{\circ}$ und war nach 6 Minuten auf $37,65^{\circ}$ gefallen. Die Temperatur der Mutter betrug 20 Minuten post partum in der Achsel $37,85^{\circ}$ und im Uterus $37,97^{\circ}$. Wenn man in Betracht zieht, dass wie Winkel *) nachgewiesen hat und wie ich speciell für diese drei Fälle bestätigen kann, die Temperatur der Mutter unmittelbar nach der Geburt mit der Tagestemperatur steigt, dass aber der Umstand, dass das Thermometer bei dem Kinde schon nach 2 Minuten seinen höchsten Stand erreicbt hatte, während diess gewöhnlich erst nach 5-10 Minuten geschieht, ein Zeichen ist, dass die Temperatur des Kindes im schnellen Fallen begriffen war, so erscheint die Annahme, dass die Temperatur des Kindes

*) Monatsschrift für Geburtskuade 1863, Bd. 22, S. 327. 
höher war als die der Mutter nicht unmotivirt *). Ein directeres Resultat ergaben die beiden anderen Messungen. Das Kind Pütz batte 3 Minuten nach Einlegung des Thermometers in den After $38,43^{\circ}$, nach 7 Minuten $38,27^{\circ}$, während die Mutter in der Achsel 15 Minuten p. p. $38,2^{\circ}, 40$ Minuten p. p. $38,3^{\circ}$ und im Uterus um 3-10 Minuten p. p. $38,2^{\circ}$ hatte. Das Kind Corte hatte unmittelbar nach der Geburt 3 Minuten nach Einlegung des Thermometers im Rectum eine Temperatur von $38,62^{\circ}$, die nach 5 Minuten auf $38,48^{\circ}$ gefallen war, während die Achselmessung der Mutter nach 20 Min. $38,35^{\circ}$, nach 25 Min. $38,45^{\circ}$ und nach 30 Min. $38,6^{\circ}$ ergab. Die Wärme des Uterus wurde, da die Placenta zögerte, nicht gemessen. Wenn schon aus diesen Messungen ebenso wie aus den Bärensprung'schen hervorgeht, dass die Ejgenwärme des Kindes im Uterus höher als die der Mutter ist, so kann ich diess noch durch einen negativen Beweis bestätigen. Während also wie oben angegeben bei Schwangern die Temperatur des Uterus die der Achsel um 0,29 und im Minimum um 0,1 übertraf, ergab bei einer Kreissenden, deren Kind 17 Stunden vorher gestorben war, für den Uterus sich nur ein Plus von $0,02^{\circ}$.

Wenn wir also für Vergleichungen mit abnormen Zuständen der weiblichen Genitalien die bei Schwangern in der Vagina gefundene Temperatur immerhin als Normaltemperatur werden benutzen können und uns dabei höchstens der Gefahr aussetzen, die Normaltemperatur etwas zu hoch anzunehmen, können wir die für den Uterus bei Schwangern gefundenen Werthe nicht als normale ansehen, werden aber jedenfalls keine grosse Fehlerquelle haben, wenn wir die Temperatur des nicht schwangern Uterus der der Vagina gleichsetzen.

Ein weiterer Umstand, der bei einem Vergleich zwischen Achsel und inneren Organen berücksichtigt werden musste, war der, dass es fraglich war, ob nicht die in inneren Organen gelegenen Thermometer die augenblickliche Körpertemperatur schneller anzeigen,

*) Nebenbei sei hier bemerkt, dass die Temperatur des allerdings nicht reifen Kindes unmittelbar nach dem Bade bis auf $\mathbf{3 5 , 4 4}$ fiel und bis zum Nachmittag 4 Stunden nach der Geburt bis auf 33,87 - im After gemessen gesunken war, ein Abfallen von $4^{\circ}$ in 4 Stunden, ohne dass das Kind irgendwie Erscheinungen von Collapsus dargeboten hätte; und erst am folgenden Morgen war die Temperatur wieder bis auf $35,3^{\circ}$ gestiegen. 
als die in der Achselhöhle gelegenen, so dass man die zu gleicher Zeit gefundenen Werthe nicht ohne Weiteres mit einander würde vergleichen können. Es war diess von grosser Wichtigkeit sowohl schon bei den einfachen Tagesschwankungen als besonders bei pathologischem Steigen und Fallen der Körperwärme. Unter normalen Verhältnissen ist diess der nur sehr langsam erfolgenden Tagesschwankungen wegen schwer $z u$ beobachten. Leichter lässt sich das Verhältniss bei abnorm gesteigerter Temperatur und schnellen Schwankungen an den Uebergängen vom Fallen zum Steigen und umgekehrt erkennen. Ich gebe in Folgendem einige Beobachtungen an einer Puerpera, deren schnelle Fieberexacerbationen von einem bedeutenden pyämischen Milzabscess, der nach der Pleuraböhle zu durchgebrochen war, herrührten.

\begin{tabular}{|c|c|c|}
\hline $\begin{array}{c}\text { Minuten } \\
\mathbf{2 0}\end{array}$ & $\begin{array}{c}\text { A. } \\
40,0\end{array}$ & $\begin{array}{l}\text { V. } \\
40,15\end{array}$ \\
\hline 40 & 39,8 & 39,9 \\
\hline 60 & 39,65 & 39,8 \\
\hline 80 & 39,4 & 39,6 \\
\hline 100 & 39,25 & 39,4 \\
\hline 120 & 39,2 & 39,37 \\
\hline 135 & 39,15 & 39,3 \\
\hline 160 & 39,13 & 39,35 \\
\hline 170 & 39,1 & 39,4 \\
\hline 180 & 39,2 & 39,4 \\
\hline 190 & 39,2 & 39,45 \\
\hline 200 & 39,2 & 39,53 \\
\hline 210 & 39,4 & 39,7 \\
\hline
\end{tabular}

Das Thermometer in der Vagina fallt constant bis zu $135 \mathrm{Mi}-$ nuten und fängt dann an zu steigen, während das in der Achsel noch bis zu 170 Min. fällt und erst dann langsam zu steigen beginnt. Umgekehrt kann es beim plötzlichen Fallen kommen, dass das Thermometer in der Achselhöhle nicht so schnell fällt, als das in der Vagina und so eine Zeit lang das erstere eine höhere Temperatur anzeigt; was sich wohl daraus erklärt, dass die dicke Haut ein schlechterer Wärmeleiter ist als die zarten Schleimhäute und so auf einen gewissen Grad erwärmt, die Wärme langsamer verliert, als letztere. Als Beispiel diene:

\begin{tabular}{|c|c|c|c|}
\hline II. & $\begin{array}{c}\text { Minuten } \\
20\end{array}$ & $\begin{array}{c}\text { A. } \\
40,2\end{array}$ & $\begin{array}{c}\text { V. } \\
40,45\end{array}$ \\
\hline & 30 & 40,4 & 40,6 \\
\hline
\end{tabular}




\begin{tabular}{cll} 
Minuten & \multicolumn{1}{c}{ A. } & \multicolumn{1}{c}{ V. } \\
40 & 40,5 & 40,75 \\
50 & $\mathbf{4 0 , 6 5}$ & 40,8 \\
60 & $\mathbf{4 0 , 8}$ & 40,8 \\
70 & 40,8 & 40,8 \\
90 & 40,65 & 40,6 \\
100 & 40,6 & 40,6 \\
110 & 40,6 & 40,6 \\
120 & 40,43 & 40,55 \\
130 & 40,35 & 40,5
\end{tabular}

wo nach 90 Minuten die Achselhöhle höher temperirt ist als die Scheide, beide dann eine Zeit lang gleich bleiben, bis schliesslich die Temperatur der Achselhöhle schnell fällt. Scheint es nach diesen Beispielen so, als ob die Vagina auf Temperaturschwankungen wirklich viel schneller reagire, so kann ich nicht verschweigen, dass nach einigen Beobachtungen das Umgekehrte der Fall zu sein scheint.

So erreicht in der folgenden Beobachtung das Thermometer in der Acbselhöhle schon nach 60 Minuten seinen höchsten Stand und bleibt darauf, während das in der Scheide erst nach 70 Minuten am höchsten steht.

\begin{tabular}{|c|c|c|}
\hline $\begin{array}{c}\text { Minuten } \\
\quad \mathbf{2 0}\end{array}$ & $\begin{array}{c}\text { A. } \\
40,4\end{array}$ & $\begin{array}{c}v . \\
40,6\end{array}$ \\
\hline 40 & 40,85 & 41,05 \\
\hline 60 & 41,15 & 41,3 \\
\hline 70 & 41,15 & 41,1 \\
\hline 80 & $\$ 1,1$ & 41,25 \\
\hline 100 & 40,9 & 41,15 \\
\hline
\end{tabular}

Aehnlich ist es in folgender Tabelle:

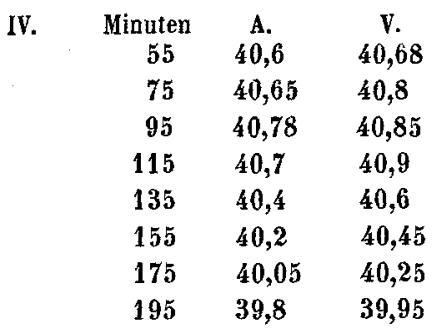

wo das Thermometer in der Achselhöhle bereits wieder fällt, während das in der Vagina erst seinen höchsten Stand erreicht. Bei weitem häufiger habe ich jedoch auch bei jähem Steigen oder 
Fallen beobachtet, dass beide Thermometer zu gleicher Zeit ihren höchsten Stand erreichten und zu gleicher Zeit zu fallen anfingen. Ich will zur Bestätigung nur einige kurze Beispiele hersetzen, die keines weitern Commentars bedürfen.

\begin{tabular}{|c|c|c|c|c|c|c|c|}
\hline \multirow[t]{6}{*}{$y$} & $\begin{array}{l}\text { Minuten } \\
20\end{array}$ & $\stackrel{\text { A. }}{40,75}$ & $\begin{array}{c}\text { V. } \\
41,0\end{array}$ & VI. & $\begin{array}{c}\text { Minuten } \\
20\end{array}$ & $\begin{array}{c}\text { A. } \\
40,6.5\end{array}$ & $\begin{array}{c}\text { V. } \\
40,8\end{array}$ \\
\hline & 40 & 41,05 & 41,3 & & 40 & 40,95 & 41,13 \\
\hline & 60 & 41,35 & 41,6 & & 60 & 41,0 & 41,2 \\
\hline & 80 & 41,55 & 41,7 & & 70 & 40,95 & 41,1 \\
\hline & 100 & 41,45 & 41,6 & & 80 & 40,95 & 41,05 \\
\hline & 120 & 41,35 & 41,45 & & 90 & 40,85 & 41,0 \\
\hline \multirow[t]{6}{*}{ VII. } & 20 & 38,55 & 38,8 & vilI. & 25 & 38,2 & 38,4 \\
\hline & 40 & 38,45 & 38,8 & & 35 & 38,1 & 38,35 \\
\hline & 60 & 38,35 & 38,63 & & 45 & 38,15 & 38,4 \\
\hline & 80 & 38,4 & 38,7 & & 55 & 38,2 & 38,45 \\
\hline & 100 & 38,5 & 38,85 & & 65 & 38,2 & 38,45 \\
\hline & 120 & 39,0 & 39,35 & & 80 & $\mathbf{3 8 , 3}$ & 38,55 \\
\hline
\end{tabular}

lch bemerke hierbei nur noch, dass ich die Anzahl dieser Beispiele bedeutend vermehren könnte und dass diess jedenfalls das gewöhnliche Verhalten ist, so dass man jedenfalls für gewöhnlich ohne bedeutende Fehlerquelle die gleichzeitig abgelesenen Temperaturen mit einander vergleichen kann und man in abweichenden Fällen durch das schnelle Steigen oder Fallen auf ein etwaiges abnormes Verhältniss aufmerksam gemacht wird.

Nachdem so diese Vorfragen erledigt, will ich zuvörderst das beobachtete Material tabellarisch zusammenstellen, um dann die für unsere Aufgabe daraus resultirenden Schlüsse zu folgern. Es betrug:

\begin{tabular}{|c|c|c|c|c|c|c|c|c|c|}
\hline & & $\begin{array}{l}\text { d. Unter- } \\
\text { schied } \\
\text { zwischen } \\
A \text { und U }\end{array}$ & $\begin{array}{l}\text { Anzahl } \\
\text { der Be- } \\
\text { obach- } \\
\text { tungen }\end{array}$ & $\begin{array}{l}\text { Anzahl } \\
\text { derVer- } \\
\text { suchs- } \\
\text { personen }\end{array}$ & $\begin{array}{l}\text { d. Unter- } \\
\text { schied } \\
\text { zwischen } \\
\text { A und V }\end{array}$ & $\begin{array}{l}\text { Anzahl } \\
\text { der Be- } \\
\text { obach- } \\
\text { tungen }\end{array}$ & $\begin{array}{l}\text { Anzahl } \\
\text { derVer- } \\
\text { suchs- } \\
\text { personen }\end{array}$ & $\begin{array}{l}\text { d. Unter- } \\
\text { schied } \\
\text { zwischen } \\
\text { U und V }\end{array}$ & $\begin{array}{l}\text { Anzahl } \\
\text { derBe- } \\
\text { obach- } \\
\text { tungen }\end{array}$ \\
\hline & Schwangeren & 0,29 & 17 & 7 & 0,101 & 55 & 20 & 0,156 & 17 \\
\hline & Kreissenden & 0,383 & 16 & 7 & 0,287 & 34 & 13 & 0,175 & 16 \\
\hline bei & Wöchnerinnen & 0,284 & 274 & 13 & 0,207 & 539 & 26 & 0,111 & 274 \\
\hline
\end{tabular}

Also erhalten wir, wenn wir dem oben auseinandergesetzten zu Folge die vom Kinde producirte Wärme bei Schwangern und Kreissenden abziehen und die genuine Uteruswärme bei Schwangern der Scheidenwärme gleichsetzen:
bei Schwangeren 0,101
0,101
0,1
bei Kreissenden $\quad 0,194$
0,287
$-0,093$
bei Wöchnerinnen 0,284
0,207
0,111 
wobei natürlich zu bemerken ist, dass der Unterschied zwischen $A$ und $\mathrm{V}$ und zwischen $\mathrm{U}$ und $\mathrm{V}$ bei Kreissenden bei weitem $\mathrm{zu}$ hoch resp. zu niedrig ausfällt, da bei erweitertem oder verstrichenem Muttermund die Eigenwärme des Fötus natürlich nicht allein die Wärme des Uterus, sondern auch die der zum Theil jetzt als Hülle des Fötus mitbenutzten Vagina bedeutend erhöhen muss. Wie viel von der für die Scheide erhaltenen Temperatur vom Fötus herstammt, entzieht sich leider der Berechnung. Die als Unterschied zwischen $A$ und $V$ bei Kreissenden erhaltene Durchschnittszahl stimmt wieder auffallend mit dem Durchschnitt, den man sich aus den Winkel'schen Angaben berechnen kann und der 0,29 bei einem Maximum von 0,47 und einem Minimum von 0,12 beträgt, während sich die betreffende Differenz im Wochenbett bei W inkel bedeutend höher nämlich auf 0,348 stellt.

Wir ersehen aber aus obiger Tabelle, dass sich bei Kreissenden die Temperatur des Uterus im Mittel um 0,093 erhöbt, eine Vermehrung der Wärme, die wir lediglich auf die Muskelthätigkeit des Organs schieben können. Ich bemerke hierbei, dass die Kreissenden sämmtlich normale Verbälinisse mit mebr weniger heftigen und schmerzhaften Wehen darboten und dass die Temperatur nur in einem Fall bis 38,6 stieg. Von einer wirklichen Entzündung konnte bei keiner die Rede sein. Ausserdem konnte die Temperatur des Uterus natürlich in den meisten Fällen nur im Anfang der Geburt gemessen werden, da bei vorrückendem Kopf das Thermometer sich nicht mehr weit genug einführen lässt. In einem Fall von Querlage betrug der Unterschied 0,6 - die höchste beobachtete Differenz, die niedrigste ist 0,15 , während in dem oben angeführten Fall, wo das Kind seit 17 Stunden abgestorben war, die Differenz nur 0,02 betrug, eine auffallend niedrige Ziffer, die sich nur durch Annabme einer bedeutenden Wärmeabgabe an die todte Masse im Innern des Uterus erklärt. Ein Wachsen der Differenz zwischen $A$ und $V$ mit der Stejgerung der Wehen liess sich nicht deutlich nachweisen, was wohl daher rührt, dass mit dem Fortgang der Geburt auch die Verhältnisse für die Wärmeabgabe Verkürzung und Klaffen der Scheide, Auseinanderlegen der Beine u. s. w. - günstiger werden. Ebensowenig konnte ich mich deutlich von dem Steigen der Temperatur während der Wehe, wie es von Winkel angegeben wird, überzeugen, obgleich ich of genug 
im Augenblick heftiger Wehen ein deutliches momentanes Steigen der Quecksilbersäule gesehen habe. Wenn man jedoch ein Thermometer zwischen zwei Finger nimmt und sieht, wie selbst durch einen schwachen Druck auf die Kugel das Quecksilber sich leicht in die Höhe treiben lässt, so lässt sich der Verdacht, dass das Steigen während der Wehe nicht auch durch den verstärkten Druck bedingt war, schwer beseitigen.

Wir haben im Obigen also ein exactes durch eine hinlängliche Anzahl von Fällen verbürgtes Beispiel einer durch blosses Functioniren bedingten Temperaturerböhung eines Organs; wie es ja nach den jetzigen Anschauungen über das Verbältniss von Wärme zur Arbeit zu erwarten war; denn vermehrter Umsatz des Stoffes liefert nie einseitig nur vermehrte Arbeit, sondern es geht, da auch die vollkommene Maschine des lebenden Organismus nicht alle Kraft in Arbeit umzusetzen vermag, ein gewisser Bruchtheil von Wärme neben der geleisteten Arbeit verloren. Die obige Wärmezunahme des Uterus bei Kreissenden bestätigt also die von Helmbolz, Heidenhain u. A. gemachten Beobachtungen an thätigen Froschmuskeln.

Interessanter und neuer ist die durch obige Zahlen bei der Rückbildung des Uterus nachgewiesene verhältnissmässig bedeutende Wärmebildung. Da jedoch unter den Wöchnerinnen eine beträchtliche Anzahl von pathologischen Fällen sich befindet, so müssen wir die einzelnen Fälle auseinanderhalten und wollen dieselben tabellarisch mit kurzer Angabe des Wochenbettverlaufes zusammenstellen: 


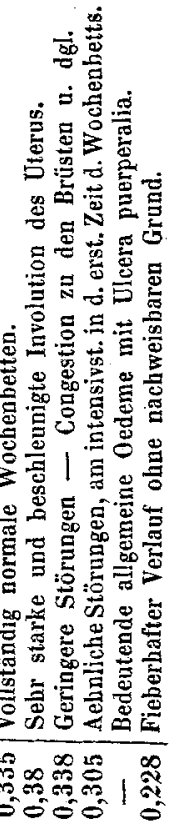

$-\infty \infty \pi+2$ is क्षणत्ब

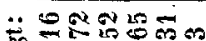

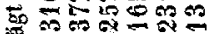

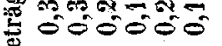
¿

口 लํำ

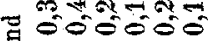

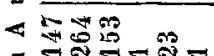

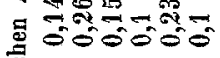

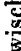

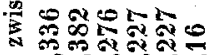

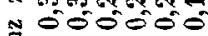
춘

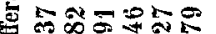
กับ 葛 क्षेठ의

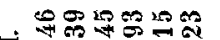
$\therefore$

o) a

$2 \operatorname{ara} a$

aो ax 4

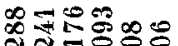
चू 42030 궁응요은 驾

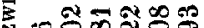
न ज० 웡 可 영엉

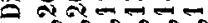
000000 $\stackrel{9}{\circ}$

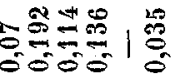
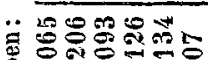

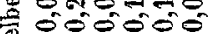
$\stackrel{8}{3}$

$\infty$ 斗 $\infty \operatorname{lo}^{+} \infty+\infty$ ग $50=0$ - 00 तn

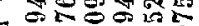
$\Rightarrow=0=0$

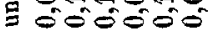
No

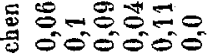
क $\frac{\infty 00000}{2000}$ 용용요 $\therefore$ 응 $=$ कीó00

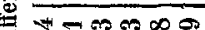
정 $0 \div-7=0$ 0ी0์00ல

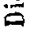

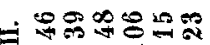

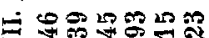

$\ddot{8}$ 离

星

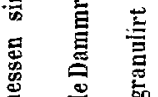

.

总要要

뭉

글

8

递

둥

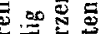

总:

要

플을

Ð 을

.

둘

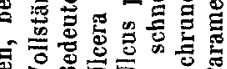

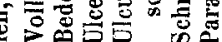

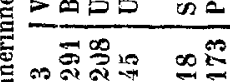

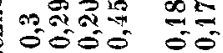

$\frac{\infty}{m a x}$

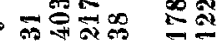

ब

500

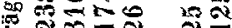

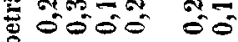

$>$ mas

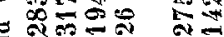

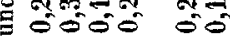

$4 \longdiv { 5 0 0 1 0 }$

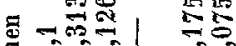

जó 0

$\infty 0^{*}+$

두요

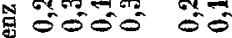

可 $=100$

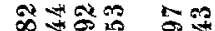

敢

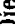

की

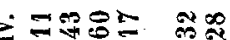
E.

and

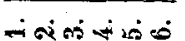

inisinis

-iकाष 
Die dritten Decimalstellen sind, weil alle Durchschnittswerthe aus einer grösseren Anzahl von Beobachtungen berechnët sind, mit notirt, ohne dass auf sie natürlich ein weiteres Gewicht zu legen wäre.

Betrachten wir nun die Resultate, die wir aus diesen Beobachtungen ziehen können und fangen wir mit dem Unterschied zwischen $\mathrm{A}$ und $\mathrm{V}$ an.

Wenn, wie wir oben gesehen haben, die Differenz zwischen A und $V$ normaler Weise die Durchschnittszahl von 0,101 gewiss nicht ubersteigt und wir im physiologischen Wochenbett im Durchschnitt eine Differenz von 0,262 oder 0,282 finden, so ist diess, obgleich ja an und für sich die Unterschiede so sehr gross nicht sind, gewiss nicht zufällig, sondern wir sehen, da diess Durchschnittszahlen aus einer grösseren Anzahl von Beobachtungen sind, deutlich, dass im Wochenbett die örtliche Wärme der Scheide im Verhältniss zu der Achsel erhöht ist. Da nun durchaus keine Gründe vorliegen, anzunehmen, dass die Temperatur der Achsel im Wochenbett erniedrigt sei - denn diese bleibt unter denselben Verbältnissen wie in der Schwangerschaft und auch vermehrte Schweisse können, wenn man die Achsel gehörig schliesst, zur Abkühlung des Thermometers gewiss nichts beitragen - so bleibt uns nur übrig, die grössere Differenz von einer bedeutenderen Wärmeproduction in der Scheide selbst abzuleiten.

Sehen wir uns nun die Verhältnisse der Scheide nach der Geburt etwas näher an. Bei jeder auch der normalsten und leichtesten Geburt ist die Scheide einer gewissen Insultation ausgesetzt, die mitunter nur in der starken Ausdehnung beim Durchtritt des Kopfes bestehen mag. Fast in allen Fällen aber erleidet die Seheide - am häufigsten der Scheideneingang - mehr oder weniger grosse wirkliche Continuitätstrennungen, die nur, weil sie gewöhnlich klein sind und von selbst heilen und weil die Scheide von dem Uterusblut überströmt wird, häufig übersehen werden. Bei 93 Wöchnerinnen, die ich kurz nach Ausstossung der Placenta auf Risse im Scheideneingang untersuchte, fand ich nur in einem Fall gar keine Verletzung, in einem andern nur blutige Sugillationen, in zweien unbedeutende Erosionen und in allen übrigen 89 Fällen fand ich deutliche Risse durch die Substanz der Schleimhaut, die allerdings in den meisten Fällen ganz unbedeutend in 
einigen Tagen, ohne die Spur einer Narbe zu hinterlassen, geheilt waren. Aber auch die Verletzungen höher in der Scheide sind nicht so selten wie man gewöhnlich annimmt. Untersucht man die Wöchnerinnen aufmerksam in den ersten Tagen des Wochenbettes, so fühlt man mitunter in der Scheide eine rauhe von der glatten Schleimhaut deutlich abgegrenzte Stelle, die man bei der Untersuchung mit dem Speculum als granulirende Fläche sieht und die zweifelsohne von einem unter der Geburt entstandenen Schleimhautriss herrührt. Wenn also geringere Verletzungen der Scheide gewissermaassen zum physiologischen Verlauf des Wochenbettes gehören, so ist es wohl gestattet, auch im normalen Wochenbett von einer entzündlichen Reizung der Scheide zu sprechen. Jedenfalls aber gehen in der Scheide einer Puerpera Prozesse vor sich, die mit der jungfräulichen Scheide verglichen, von einem bedeutend gesteigerten Stoffwechsel Zeugniss geben. Während in der Schwangerschaft nicht blos der Uterus, sondern auch die Vagina an Masse bedeutend zugenommen hat, während auch die Muskelschichten in den Scheidenwänden verdickt sind und das ganze Gewebe der Scheide hypertrophisch geworden ist, geht im Wochenbett eine allmähliche Rückbildung des Organs vor sich. Eben so wie wir die Epithelien massenhaft abgestossen im Lochialsecrete finden, werden die dicken Muskelbündel - wie bei der Involution des Uterus zur fettigen Degeneration und zur Resorption gebracht und die Scheide nimmt - allerdings nur annähernd - ihre alten Verhältnisse wieder an. Wenn mun von den heutigen Physiologen als alleinige thierische Wärmequelle die 0xydation organischer Substanzen angesehen wird, so werden die Schlüsse gewiss nicht gewagt erscheinen, dass das Plus der örtlichen Wärme in der puerperalen Scheide durch die energische Rückbildung der Gewebe erzeugt wird, die ja ohne schnelle Oxydation und allmähliche Ueberführung böherer organischer Verbindungen in immêr niedrigere nicht denkbar ist und dass bestehende örtliche Entzundungen, in denen der Stoffwechsel noch beschleunigt ist, die Höhe dieser Wärmeproduction nur steigern können.

Ist diess richtig, so werden wir bei Primiparen, deren Scheide noch nicht ausgedeknt gewesen ist und die deswegen, wie sich statistisch nachweisen lässt, leichter Verletzungen ausgesetzt sind, einen grösseren Unterschied erwarten können als bei Multiparen, 
und dieser Umstand erklärt den Unterschied zwischen Tabelle II. und IV., denn die Messungen im Uterus selbst wurden, da die sichere Einführung des Thermometers in den Uterus nicht ohne einen gewissen Druck gegen das Perineum geschehen kann, nur bei solchen Wöchnerinnen gemacht, die geringe Verletzungen im Scheideneingang hatten, so dass unter den 13 betreffenden Versuchspersonen nur 4 Primiparae sind, während unter den 9 der Tab. IV. nur 2 Multiparae sind. So beträgt denn der aus Tab. VI. berechnete Gesammtunterschied zwischen $\mathrm{A}$ und $\mathrm{V} 0,178$, der aus Tab. IV. berechnete 0,23 ; und scheiden wir die beiden Klassen vollständig, so beträgt die mittlere Durchschnittszahl bei Mehrgebärenden in den ersten $17 \times 12$ Stunden 0,195 , während sie bei Erstgebärenden 0,257 beträgt. In der Tab. IV. haben wir 3 Personen, bei denen der mittlere Unterschied den des normalen Wochenbetts übersteigt und in allen 3 Fällen haben wir Dammrisse, die zum Theil per primam int., zum Theil durch üppige Granulationen heilen.

Ein ähnliches nur stärker ausgeprägtes Verhältniss wie zwischen $\mathrm{A}$ und $\mathrm{V}$ finden wir $\mathrm{zwischen} \mathrm{A}$ and $\mathrm{U}$. Während, wie wir oben gesehen haben, die Differenz zwisehen beiden normaler Weise nicht über 0,101 betragen kann, sehen wir dieselbe im physiologischen Wochenbett auf 0,337 erhöht, ein Umstand, der nicht auffallen kann, wenn wir die schnelle und energische Involution des Uterus, die nur durch massenhafte Verbrennung seiner organischen Bestandtheile möglich ist, in Betracht ziehen. In Uebereinstimmung damit sehen wir denn diese Differenz bei sehr energischer Ruickbildung bis auf 0,38 erhöht.

Es ist also durch diese Ziffern, die an und für sich nur klein, doch als das Mittel aus einer grösseren Anzahl unter sich übereinstimmender Beobachtungen alle Beachtung verdienen, die wichtige Thatsache, gegen die theoretischerseits gewiss nichts einzuwenden ist, auch durch die Beobachtung constatirt, dass nicht blos bei Entzündungen, sondern auch bei einfacher physiologischer Rückbildung eines 0rgans örtlich Wärme producirt wird; und wenn man einen Schritt weiter geht, so kann man, da jede Entzündung aus Anbildung und Rückbildung besteht, die Assimilation der zugeführten Stoffe zum Zelleninhalt (s. unten) aber nicht wohl von Wärmeentwickelung begleitet sein kann, nicht ohne Grund sagen, 


\section{1}

dass auch bei der Entzündung die Wärmeentwickelung von der regressiven Stoffmetamorphose ausgeht.

Halten wir diesen Gesichtspunkt fest und sehen wir uns an der Hand obiger Beobachtungen die Theorie der organischen Wärmebildung etwas näher an.

Wenn wir mit der einfachen Hyperämie beginnen, so ist die Frage, ob mit der Hyperämie eine örtliche Temperatursteigerung verbunden sei, auf das Vielseitigste ventilirt und meines Wissens ist diese Frage immer dahin beantwortet, dass die Steigerung der Temperatur, die man dabei beobachtet, lediglich von einer grösseren Füllung der Gefässe abhängt. Diese Art der Temperatursteigerung ist aber eine vollständig nebensächliche und überhaupt wohl nur in wenig vascularisirten, peripherisch gelegenen und deshalb einer starken Abkühlung ausgesetzten Organen möglich. Die meisten innern Organe sind so reichlich von Blut durchströmt und von einem so feinen Capillarnetz durchzogen, dass man ihre Temperatur der Bluttemperatur wird gleichsetzen können. Ausserdem ist die hauptsächliche Wärmequelle unter normalen Verhältnissen nicht im Blut, sondern in den Zellen selbst zu suchen und der schnelle Kreislauf des Blutes tauscht die gewiss nicht überall in gleichem Maasse producirte Wärme so vollkommen aus, dass normaler Weise nur ein geringer Unterschied messbar wird. Die Temperatur der meisten innern Organe wird also, da die Verbrennungsprocesse in den Geweben selbst stattfinden, annähernd der Temperatur des Blutes der tiefer gelegenen Venen gleich steben. Bestätigt wird diese Anschauung durch die von John Simon und 0 . Weber*) gemachten thermoelectrischen Messungen an entaündeten Theilen, die beide die Entzündungsstelle wärmer fanden als das zu- und abfliessende Blut, immer aber die Vene wärmer als die Arterie, denn dass sie unter pathologischen Verhältnissen z. B. die Cruralvene regelmâsssig kälter fanden als den Entzündungsheerd, kann, da das von demselben kommende Blut durch Vermischung mit dem Blute der ganzen Extremität bereits etwas abgekiuhlt sein musste, nicht befremden. Wenn man somit annehmen muss, dass die Temperatur der meisten inneren Organe von der

*) Handbuch der allgemeinen a. spec. Chirurgie von Pitha und Billroth. Bd. 1. S. 385 . 
Bluttemperatur nicht wesentlich unterschieden ist, so kann ihre Temperatur dadurch, dass noch mehr Blut von glejeher Wärme in sie strömt, natürlich nicht erhöht werden. Anders gestaltet sich die Sache allerdings bei wenig vascularisirten, peripherisch gelegenen und deswegen der Abkühlung leicht ausgesetzten Theilen. Als Beispiel, wie sehr die blosse Hyperämie die Temperatur eines Theils erhöhen kann, wird in allen Lehr- und Handbüchern das bekannte Bernard'sche Experiment der Sympathicusdurchschneidung angegeben. Ich habe den Versuch einige Male nachgemacht und will, da mir die erhaltenen Resultate immerhin interessant zu sein scheinen, dieselben hier kurz anfühıren. Ich bemerke dabei, dass Kaninchen überhaupt zu thermometrischen Messungen wenig geeignet sind, da ihre Körperwärme sehr schnellen und grossen Schwankungen unterworfen ist. So habe ich - wie schon Kussmaul und Tenner - die Temperatur im Rectum bei festgebundenen unverletzten Thieren bei einer Zimmerwärme von c. $20^{\circ}$ R. in einer Stunde um $2^{\circ}$ fallen sehen. Den obigen Versuch machte ich, indem ich das Thier festband, den Leib, um möglichst die Abkühlung zu verhindern, mit Watte bedeckte, ein Thermometer in das Rectum - stets mindestens 6 Centim. tief - einführte und zwei andere in die Obren legte. Jedes Ohr wurde um das drinsteckende Thermometer gewickelt, um das Ohr Watte gelegt und diese um jedes Ohr einzeln und dann um beide Ohren zusammen festgebunden, so dass die Abkühlung möglichst aufgehoben war. Ich fand dabei in Versuch I. vor dem Experiment

$\begin{array}{ccc}\text { Rectum } & \text { Rechtes ohr } & \text { Linkes } 0 \mathrm{hr} \\ \mathbf{3 9 , 0} & \mathbf{3 8 , 7 2} & \mathbf{3 8 , 3}\end{array}$

Nachdem der Sympathicus der rechten Seite, während sämmtliche Thermometer liegen bleiben, durchschnitten war, sank die Temperatur des Mastdarms, die schon vorher im Fallen war, weiter, die Temperatur des rechten Ohrs fiel anfangs gleichfalls noch etwas, jedoch sehr Jangsam und blieb nach einer Stunde mit geringen Schwankungen stehen, während die Temperatur des linken Ohrs schnell fiel. So war nach $2 \frac{1}{2}$ Stunden, als der Versuch abgebrochen wurde, die Temperatur des Rectum 38,45, die des rechten Ours 38,7 , die des linken 33,73. Bei einem andern Versuch war das Verbältniss folgendes: 


\begin{tabular}{|c|c|c|c|c|}
\hline II. & Rectum & Rechtes $0 \mathrm{hr}$ & Linkes $0 \mathrm{hr}$ & \\
\hline ing . & $\mathbf{3 8 , 8}$ & 37,3 & 37,15 & \\
\hline Stund. nach der Durchschneid & 37,8 & 35,95 & 37,0 & archschnitten. \\
\hline
\end{tabular}

Bei einem dritten Versuch war die Temperatur: III. Rectum Rechtes Ohr Linkes Ohr Vor der Durchschneidung . . $37,95 \quad 37,15 \quad 37,61$ Rechter Sympathicus 2 Stund. nach der Darchschneid. 36,9 37,2 35,2 durchschnitten.

Bei einem vierten:

IV. Rectum Rechtes $0 \mathrm{br}$ Linkes $\mathrm{Obr}$ 10 Min. nach der Durchschneid. $39,3 \quad 38,65 \quad 37,84$ Rechter Sympathicus $3 \frac{1}{2}$ Stund, nach der Durchschneid. 38,55 38,27 33,6 durchschnitten.

Man sieht, dass allerdings die Temperatur des durchschnittenen Ohrs im Verhältniss zu der des Rectum steigt, dass aber die Hauptdifferenz zwischen den beiden Ohren durch das Fallen der Temperatur im $\mathrm{Ohr}$ der nicht durchschnittenen Seite bedingt ist, was wohl durch eine vom centralen Theil des durchschnittenen Sympathicus durch das Rückenmark hindurch auf den Sympathicus der andern Seite übertragene Reizung zurückzuführen ist. Für unsere Zwecke interessanter ist der Umstand, dass in Versuch I. und III. die Temperatur des Ohrs der durchschnittenen Seite die des Rectum im ersten Fall um 0,25, im andern Fall um 0,3 übertraf. Diese absolute Temperaturerhöhung war nicht vorübergehend, sondern wurde im ersten Fall 2, im andern 1 Stunde lang beobachtet und dauerte, als der Versuch abgebrochen wurde, in beiden Fällen noch fort. Wenn man nun auch grade bei Kaninchen mit der Verwerthung thermometischer Messungen vorsichtig sein muss, so glaube ich doch, dass selbst das in Watte dicht eingehüllte Ohr die Quecksilberkugel nicht so vollständig zu umschliessen vermag, wie der After. Wenn also dessenungeachtet das Thermometer im Ohr höher steht, als das 6 Centim. in den After eingeführte Thermometer, so glaube ich diese $0,25^{\circ}-0,3^{\circ}$ auf Rechnung örtlich in dem $\mathrm{Ohr}$ entstandener Wärme bringen zu dürfen.

Dass Kussmaul und Tenner*) diese absolute Temperaturerhöhung bei ihren Experimenten nicht nachweisen konnten, liegt jedenfalls einerseits darin, dass sie, wie es scheint, das Thermometer im Rectum nicht liegen liessen, so dass sie nicht in jedem Augenblick die Temperaturen unter einander vergleichen konnten,

*) Molesch ot t's Untersuchungen. 1856. Erster Band. S. 96.

Archiv f. pathol. Anat, Bd, XXXV, Hft. 2. 
andererseits aber darin, dass ihre Methode, die Temperatur des Ohrs zu messen, keine fehlerfreie zu nennen ist. Denn immer ist das einfach um die Thermometerkngel gewickelte Kaninchenohr durch die nicht ausgeschlossene Verdunstung und durch die geringe Dicke seiner deckenden Wandungen in Bezug auf die Erwärmung des Quecksilbers sehr ungünstig gegen das Rectum situirt, was auch schon daraus hervorgeht, dass Kussmaul und Tenner in den meisten Fällen bedeuteud böhere Differenzen zwischen $0 \mathrm{hr}$ und Rectum fanden, als ich bei meinen Versuchen, wo durch sorgfảltigen Watteverschluss diese ungünstigen Momente möglichst beseitigt waren. Wenn nun dessenungeachtet bei Kussmaul und Tenner die Differenz mitunter sehr gering wurde, in meinen Versuchen aber zweimal zu Gunsten des Obrs ausfiel, so verdient diess volle Beachtung. Auch von theoretischer Seite kann man, wie ich glaube, gegen die gesteigerte Warmeproduction bei der $\mathrm{Hy}_{-}$ perämie keine Schwierigkejten erheben. Wenn die normale Wärme durch den normalen Stoffwechsel producirt wird, so muss bei gesteigertem Stoffwechsel auch die Wärmeproduction gesteigert sein. In der Hyperämie - ich spreche hier fortwährend nur von der arteriellen - sehen wir aber auf das Evidenteste den Stoffwechsel beschleunigt. Die Gefässe sind nicht bloss stärker gefüllt, sondern der Kreislauf geht auch bei weiteren Gefässen mit Verringerung der Widerstände schneller von statten und mit der gesteigerten Zufuhr von Ernährungsmaterial und von Sauerstoff geht auch eine Steigerung der Zellenernährung und der Oxydation vor sich. „Es ist," sagt Weber (a. a. 0. S. 51), "zweifellos, dass anhaltende oder oft wiederholte Hyperämien zur Hypertruphie Veranlassung geben können, wie dies schon das gewöhnliche Wechselverhältniss zwischen der häufigen Thätigkeit eines Organs und seiner stärkeren Entwicklung beweist," und erinnert dabei an die zahlreichen Hypertrophien in Folge oft wiederholter functioneller Hyperämie. Gewiss aber kommt die Hypertrophie eines Organs nicht durch blosse Vermebrung der Anbildung zu Stande, sondern daneben findet jedenfalls immer eine erhöhte regressive Metamorphose statt, so dass nicht die A:fnahme von Ernährungsmaterial einseitig vermehrt ist, sondern dass überhaupt der ganze Stoffwechsel beschleunigt ist, nur dass im Ganzen ein Minimum mehr zum Aufbau verwandt wird, als in oxydirter Form das Organ verlässt, so dass allmählich 
durch das geringe aber andanernde Ueberwiegen der Anbildung das betreffende Organ eine Massenzunahme erfährt.

Unklar und von den Physiologen wenig berücksichtigt ist allerdings noch die hier bedeutend in Betracht kommende Rolle, die der Aufbau von Zellen und die Assimilation des Ernährungsmaterials zum Zelleninhalt in dem thierischen Wärmehaushalt spielt. Gewiss wird bei diesen Vorgängen, wo jede Oxydation wegfällt, keine Wärme frei; ob bei dem Aufbau und der Ernährung der Zellen Wärme gebunden wird, ist eine andere Frage. Wenn aber auch die Fortschritte der Wissenschaft diese bejahend beantworten sollten, so wird man daraus kein Moment gegen die örtliche Wärmebildung bei der Hyperämie entnehmen können; denn unser Nahrungsmaterial wird uns in hoch organisirten Verbindungen zugeführt und verlässt den Organismus in den niedrigsten Oxydationsstufen, so dass jedenfalls bei dem rückbildenden Vorgang eine unverhältnissmässige Wärmemenge gegen die etwa bei dem anbildenden verbrauchte frei werden muss.

Die Gründe nun und die Experimente, die Kussmaul und Tenner*) gegen die Wärmeproduction bei Durchschneidung des Sympathicus in's Feld fübren, sind vorzugsweise gegen die Bernard'sche Ansicht eines unmittelbaren Einflusses des Sympathicus anf die Wärmebildung gerichtet und fallen zum Theil weg, wenn man statt durch Durchschneidung des Sympathicus entstandene $\mathrm{Hy}-$ perämie überhaupt jede Hyperämie setzt. Denn dann versteht es sich von selbst, dass sie bei collateraler Hyperämie, die ja nach Unterbindung der beiden A. subclaviae und einer A. carotis in der andern Carotis sebr bedeutend sein musste, eine gleiche oder gar höhere Temperatur als nach der Durchschneidung des Sympathicus erhielten. Dass aber in dem Ohr der durchschnittenen Seite nach Unterbindung der zuführenden Gefässe bei fehlender oder mangelhafter Herstellung des Collateralkreislaufes die Wärme abnehmen musste, ist einleucbtend. Denn wenn auch (S. 100) die Gefässe bei ihren Versucben nie ganz blutleer wurden, so war doch eben die Zufuhr vou Sauerstoff aufgehoben, und wenn überhaupt, wie Kussmaul und Tenner meinen, in dem die Gewebe durchtränkenden Näbrsaft freier Sauerstoff sich befindet, was durchaus nicht

*) a. a. 0. S. 100 . 
wahrscheinlich ist, so ist dieser doch keinesfalls in solcher Menge da, dass er auch nur die gewöhnliche Quantităt Wärme produciren könnte. Ja, der Sauerstoff an und für sich ist zur Oxydation organischer Substanzen durchaus nicht geeignet, so dass nach Sehönbein Thiere ohne Blutköpperchen mitten im Sauerstoff ersticken, sondern er muss in Ozon umgewandelt sein und es ist desshalb zur gehörigen Wärmebildung die Zufuhr von rothen Blutkörperchen als Ozonerregern durchaus nothwendig; eine Ansicht, die bestätigt wird durch die Versuche von Pokrowsky*), der constant durch Eisenpräparate - gleichfalls Ozonbringern - die Körpertemperatur und die Harnstoffausscheidung zu erhöhen vermochte. Dass auch der ausgeschnittene, von allem Blut befreiete Froschmuskel nach Helmbolz Wärme entwickelt, lässt sich, da er der atmosphärischen Luft, in der stets etwas freies Ozon ist, ausgesetzt ist, und da die Oxydation durch die Gegenwart der alkalischen interstitiellen Gewebsflüssigkeiten erleichtert ist, ungezwungen erklären. Die noch in jeder Beziehung dunkle Wärmeentwicklung nach dem Tode kann aber, da sie ein noch ganz unaufgeklärtes und räthselhaftes Phänomen ist, als Gegenbeweis nicht benutzt werden. Wenn somit die genauen und mühsamen Versuche obiger Forscher gegen die Wärmebildung bei Hyperämie nichts beweisen, ja dieselbe sogar wahrscheinlich machen, so liegen uns eine Menge anderer Thatsachen vor, die sich für dieselbe anführen lassen. Die gewöhnlichste Art der Hyperämie ist die functionelle Fluxion, und wir sehen auf das Evidenteste bei jeder Fluxion auch die Wärme vermehrt. Dieselbe lässt sich allerdings, da sie durch eine Menge entgegenwirkender Umstände -- die sehr bedeutende Wärmeabgabe durch Verdunstung u. s. w. - zum grössten Theil paralysirt wird, durch das Thermometer nur in sehr beschränkter Art nachweisen. Weit leichter gelingt diess durch die Calorimetrie. So fand beispielsweise Hirn, dass ein 42 jähriger Mann, der in der Ruhe 149000 Calorien producirte, bei Bewegung es auf 275000 brachte, ein sehr bedeutender Unterschied, wenn man bedenkt, dass diess Plus allein durch Muskelfunction bedingt wurde. Jede Function ist aber mit einer fluxionären Hyperämie verbunden und nur durch die vermehrte Sanerstoffzufuhr wird die Wärmeentwicklung ermög-

*) Dieses Archiv Bd. XXll. S. 476 u. f. 
licht. Wenn aber schon bei der functionären Hyperämie neben der geleisteten Arbeit eine so bedeutende Wärmemenge producirt wird, so muss bei jeder andern Hyperämie, die keine Arbeit zu leisten hat, in Folge des Wechselverhältnisses zwischen Wärme und Arbeit die gelieferte Wärme eine viel bedeutendere sei.

Wenn somit anzunehmen ist, dass bei jeder das normale Maass übersteigenden Blutzufuhr auch eine das normale Maass übersteigende Wärmemenge gebildet wird - die allerdings in sehr vielen Fällen der zugleich stattfindenden Vermehrung der Wärmeausgaben wegen durch Thermometrie kaum nachzuweisen ist - so muss man a priori zugeben, dass diese Wärmemenge bei der Entzündung, wo der rückbildende Prozess den anbildenden übertrifft, eine um so grössere sein muss. Dieses Ueberwiegen des riickbildenden Processes ist das für die Entzündung der Hyperämie gegenüber einzig Charakteristische. Einen wirklich principiellen Unterschied aufzustellen, ist unmöglich, und schon Virchow*) kommt zu dem Schluss, dass die ontologische specifische Auffassung der Entzündung aufzugeben sei. Er lässt sie von den andern Ernährungsstörungen sich nur durch die Grösse ihrer Leistung, dureh die Schmelligkeit ihres Verlaufs, bauptsächlich aber durch den Charakter der Gefahr unterscheiden. Die beiden ersteren Merkmale sind aber nur graduelle, das letztere, auf das er das meiste Gewicht legt, ist ein von dem Ausgang des Prozesses hergenommenes und kann, wenn es auch von eminenter practischer Bedeutung ist, doch keinen principiellen Unterschied bedingen. So sehen wir auch täglich Hyperämie in Entzündung übergehen; man kann beide Processe, wo sie ausgesprochen sind, hinlänglich unterscheiden, Niemand aber kann im gegebenen Fall den Moment bestimmen, wo die Hyperämie aufhört und die Entzündung anfängt. Deutlich charakterisirt ist die letztere erst, wenn die Bilance der Einnahmen und Ausgaben zu Gunsten der Ausgaben gestört ist, wenn die Ernährung der alten und die Production neuer Zellen gegen die degenerative Metamorphose zurückbleibt, wenn der Anbau des zugefübrten organischen Materials zum functionsfähigen Zelleninhalt gegen den Zerfall des letzteren in höhere Oxydationsstufen, d. h. in niedrigere organische Verbindungen zurücktritt. Einleuchtend ist

*) Handbuch der speciellen Pathologie und Therapie. 1854. Erster Band. S. 76. 
dabei, dass mit diesem Ueberwiegen der oxydationsprozesse die örtliche Wärmebildung bedeutend zunehmen muss.

Es wird aber dieser theoretische Schluss, dass jede Entzündung mit Wärmeentwicklung verbunden ist, durch eine grosse Zahl Beobachtungen bestätigt. Auch in unsern oben angeführten Tabellen haben wir die unzweideutigsten Beweise dafür. Bei heilenden Dammrissen (s. Tab. IV. Nr. 2) war die Temperatur der Scheide in den ersten $17 \times 12$ Stunden 0,364 höher als die der Achsel, während der Unterschied normaler Weise höchstens 0,278 beträgt. Sehr interessant ist in dieser Beziehung der von mir in 204 Fällen angestellte Vergleich der Temperatur des Scheideneingangs mit der des Scheidengrundes. Während ich bei Schwangern fast ohne Ausnahme beide gleich warm und nur in sehr wenigen Fällen den Scheideneingang etwas kühler antraf, fand ich im normalen Wochenbett bei fehlenden Rissen im Scheideneingang im Durchschnitt diesen 0,15 , bei unbedeutenden Verletzungen 0,08 kälter als den Scheidengrund; bei gut aussehenden heilenden Verletzungen fand ich den Scheideneingang 0,07 wärmer, bei schlecht eiternden, schlaff aussehenden Geschwürsflächen hingegen ihn wieder 0,12 kälter als den Scheidengrund; ja es liess sich in einzelnen Fällen der Bereich der frischen Entzündung im Scheideneingang Zoll für Zoll mit dem Thermometer nachweisen. In einem Fall wandelte sich ein ziemlich bedeutender seitlicher Schleimhautriss in ein grosses Geschwür um, das im Durchschnitt 0,08 kälter war als der Scheidengrund, als jedoch vom 8 . Tage an dasselbe zu heilen begann, zeigte es $0,06 \mathrm{mehr}$, während vom 12 . Tage an, als es ziemlich geheilt war, keine erhebliche Differenz mebr nachzuweisen war.

Während hierdurch einerseits dic älteren Beobachtungen uber Wärmeproduction bei Entzündung bestätigt werden, konnte ich in Fällen, wo die Schleimhautrisse statt zu heilen sich in schlecht aussehende, schlaff granulirende Geschwürsflächen verwandelt hatten, in keinem Fall eine über die Norm erhöhte örtliche Wärmeproduction nachweisen; ja in allen diesen Fällen blieb das Verhältniss zwischen $A$ und $V$ hinter dem normalen zurück. Auch nach den Winkel'schen Messungen wurde die niedrigste Differenz zwischen $A$ und $V 0,284$ (gegen 0,392 im normalen Wochenbett) bei enorm grossen Geschwüren im Scheideneingang angetroffen. Ein 
ganz ähnliches Resultat, wie wir es erhalten haben, geht aus den Weber'schen Versuchen *) hervor. Bei seinen Versuchen am Menschen ergab allerdings die erste Messung, obgleich einen alten Abscess betreffend, ein Plus gegen die Mundhöhle. Die Temperatur dieser letzteren ${ }^{* *}$ ) ist aber zu wenig constant und zu ungünstigen Einflüssen ausgesetzt, als dass diese Messung etwas beweisen könnte. Etwas anderes ist es in den beiden folgenden Versuchen, in denen er verwundete Backen mit den gesunden verglich und in denen er bei frischer Entzündung ein Plus für die Wunden erhielt. In Versuch $\mathrm{E}$ maass er "ein seit mehreren Monaten bestehendes tiefes Fistelgeschwür, das die Messung daher leicht machte, " und fand in der Fistel 37,1, in der Achselhöhle 37,2. Weber bemerkt gleichfalls bei dieser Gelegenheit, dass nur in den ersten Tagen die Differenz zu Gunsten der Wunde merklich ist, dass sie später abnimmt und bei längerer Eiterung zu Gunsten des Blutes ist. Messungen an Kaninchen sind, wie ich schon oben bemerkt habe, wenig zuverlässig, indess ist auch hier bei dem ersten Versuch bei stärkerer Eiterung die Differenz zwischen links und rechts $=0$, während sie sonst ein bedeutendes Plus zu Gunsten der entzündeten Seite gibt, ein Resultat, mit dem allerdings der 6. am Kaninchen angestellte Versuch in directem Gegensatze steht. Dass Weber's bezügliche Versuche an Hunden zum grossen Theil negative Resultate ergaben, kann, da er die Temperatur von Extremitäten mit der Aftertemperatur verglich, nicht befremiden, doch ergaben auch hier einzelne und besonders häufig die genaueren thermoelectrischen Untersuchungen ein Plus für die Wunde.

Es scheint somit, als ob nur die frische reactive Entzündung wirklich Wärme erzeugt und als ob ältere und besonders eiternde Wunden die örtliche Temperatur wenig oder gar nicht steigern; und es lässt sich diess sowohl bei schlecht aussehenden schlaffen Geschwüren, wo eben eine eigentliche Entzündung fehlt und wo der Stoffwechsel gewiss nicht beschleunigt ist, als auch bei starker Eiterung begreifen. Denn bei letzterer fliessen die massenhaft neugebildeten Zellen, statt ferneren Oxydationsvorgängen zu

*) Deutsche Klinik 1864. No. 43 u. 44.

**) Kliniscbe Untersuchungen u. s. w. von Liebermeister. Prager Vierteljahrsschrift. 1865. Erster Band. S. 11. 
verfallen, nach aussen $\mathbf{a b}$ und binden durch die Verdunstung der sie führenden serösen Massen höchstens Wärme, wenn nicht die auffallend geringen Differenzen zwischen $A$ und $V$, die ich unter diesen Verhältnissen erhielt, vielleicht auf den oben als hypothetisch angedeuteten Wärmeconsum bei der Zellenbildung hinweisen.

Wenn wir also als constatirt annehmen können, dass bei frischer Entzündung örtlich Wärme erzeugt wird, so kommen wir jetzt zu dem schwierigen Verbältniss zwischen der örtlichen Wärmeproduction und der Steigerung der allgemeinen Körperwärme, d.h. zu dem Verhältniss zwischen Entzündung und Fieber. Von vornherein klar ist die Thatsache, dass ein Plus von Wärme an irgend einem Orte auf die Allgemeintemperatur einen Einfluss haben muss und nur uber die Bedeutung dieses Einflusses differiren die Meinungen. Während Zimmermann*) die Bedeutung der örtlichen Wärmeproduction für das Fieber vollständig in den Vordergrund stellt und das letztere einzig und allein und unter allen Umständen nicht bloss von einer bestehenden Entzündung abhängig macht, sondern auch ungefäbr die ganze Fieberböhe von der örtlich producirten Wärme herleitet, leugnet Billroth**) überhaupt die Wahrscheinlichkeit, dass in einem entzündeten Theil ein auf die Erwärmung der gesammten Blutmasse messbar einwirkende Wärmemenge erzeugt wird. Er führt, um diesen Satz plausibel zu machen, ein nach meiner Ansicht nicht glücklich gewähltes Beispiel (S. 373) an. Der Vergleich mit einem kleinen Ofen, der ein Zimmer von constanter Temperatur nicht schnell erwärmen kann, möchte passend erscheinen, wenn man sich die Circulation des Blutes wegdenkt, so dass die Wärme sich durch den Körper wie durch die Luft des Zimmers lediglich durch Leitung und durch Strablung verbreiten könnte. In dem Blutumlauf haben wir aber ein weit vollkommneres und schnelleres Communicationsmittel, durch das es auch einer verhältnissmässig geringen aber constant erzeugten Wärmemenge ermöglicht wird, auf den Gesammtkörper einzuwirken. Denn das in dem Wärme producirenden Organ befindliche Blut wird wenigstens annähernd auf die in dem Gewebe herrschende

*) Deutsche Klinik 1862. No. 1, 41, 44. 1863. No. 43-49. 1864. No. 32, 34, 35. 1865. No. 27, 28, 30.

*) Langenbeck's Archiv 1864. 2. Heft. S. 381 . 
Temperatur erwärmt und da fortwährend neues Blut hindurchströmt und alles durchströmende Blut das betreffende Organ wärmer verlässt, so muss bei fortdauernder Wärmeproduction selbst eine unbedeutende Wärmequelle auf die Steigerung der Allgemeintemperatur einen Einfluss haben. Dieser Einfluss der örllichen Wärme zeigt sich in den obigen Tabellen auf das deutlichste. Die Differenz zwischen $A$ und $V$ ist am grössten Tab. IV. No. 2. bei bedeutender örtlicher Entzündung der Scheide, geringer ist sie unter normalen. Verhältnissen, noch geringer wird sie, sobald die Körperwärme von einem anderen Heerde aus über die Norm erhöht wird. So vermindert sich Tab. I. No. 2. dadurch, dass die im Uterus producirte Wärme wächst und die Temperatur des ganzen Körpers erhöht, naturgemäss der Unterschied zwischen Achsel und Vagina, während der zwischen Uterus und Vagina sich ganz bedeutend über die Norm erhebt. So wird bei abnormer Wärmeproduction in anderen Organen (Brüste, Peritoneum) die Differenz zwischen Achsel und Uterus, ebenso wie die zwischen Achsel und Vagina geringer,' während die zwischen Uterus und Vagina sich nicht erheblich verändert, jedenfalls aber nicht kleiner wird. Sehr deutlich sieht man diesen Einfluss in Tab. IV. No. 5., wo eine Primipara in den ersten 8 bis 12 Stunden mindestens das normale Verhältniss zwischen Acbsel und Vagina zeigt, das sich aber von da an, wo eine andere Wärmequelle - Schrunden an den Warzen - die allgemeine Temperatur steigert, so ändert, dass die Differenz $z$ wischen Achsel und Vagina, statt in dieser Zeit des Wochenbetts zu steigen, bedeutend sinkt.

So finden wir auch von allen Autoren angegeben, dass selbst gesteigerte Functionen einzelner Organe die Allgemeintemperatur steigern, und zwar nimmt die Körpertemperatur während stärkerer Muskelanstrengung nach Dury um $\frac{1}{3}$ bis $\frac{3}{4}^{\circ} \mathrm{C}$. zu. In der Wirklichkeit ist aber die Vermehrung der Wärme, wie die schon oben angefübrten calorimetrischen Versuche von Hirn ergeben, viel bedeutender und es wird nur für gewöhnlich unter normalen Verhältnissen das bedeutende Plus der erzeugten Wärme durch compensirende Einrichtungen zum bei weitem grössten Theil nach aussen abgegeben. Während in sehr heissen Räumen*) bei un-

*) Vierordt, Grundriss der Physiologie des Menschen. 1864. S. 248. 
geheuer gesteigerter Schweissbildung die Temperaturerhöhung höchstens 1 bis $2^{\circ}$ beträgt, steigt sie in einer mit Wasserdämpfen überladenen Luft, welche die Verdunstung einigermaassen verhindert - iḿ russischen Dampfbad - schnell um 2 bis $3^{0}$ und Liebermeister (a. a. 0. S. 45) vermochte dureh ein Bad von Körperwärme, also durch einfache noch nicht einmal vollständige Verhinderung der Wärmeabgabe die Temperatur in $55_{\frac{3}{4}}^{3}$ Minuten um $1,27^{\circ}$ zu steigern. Aus den für die Wärmeabgabe günstigen Verbältnissen Gesunder erklärt es sich auch, warum Schwangere, die, wie wir oben gesehen haben, in dem Kinde eine fortwährende Wärmequelle in sich tragen, nicht eine abnorm gesteigerte Temperatur zeigen - aus ihnen erklär't es sich, dass die Wöchnerunnen, die in der Milch und den Lochien eine bedeutende Menge nicht vollständig oxydirter Verbindungen abgeben und die durch die den Wochenbett eigenthümliche starke Schweisssecretion gewiss viel Wärme verlieren, trotz der Involution des Uterus und den entzündlichen Reizungen der Scheide eine mässige Körperwärme zeigen. Dass aber auch unter vollständig normalen Verhältnissen die Körperwärme bei Wöchnerinnen über das sonst als normal angenommene Maximum steigen kann, zeigt die tägliche Erfahrung. Denn nicht selten sieht man bei Wöchnerinnen, die durchaus nichts pathologisches darbieten, an einzelnen Abenden die Temperatur bis über $38,0^{\circ}$ steigen, ja ich glaube, dass man in vielen Fällen, wo die Temperatur selbst bis 39,0 und etwas darüber steigt und sonst alle Verbältnisse normal sind, diese Steigerung getrost der Involution des Uterus zuschreiben kann. Zu dieser Auffassung ermuthigt mich hauptsächlich der eine von den unter Tab. I. No. 2. mitgetheilten Fällen. Die Multipara, bei der der Uterus durch die Wendung seines Inhalts entledigt war, hatte die heftigsten Nachwehen bei steinhart sich anfüblendem Uterus. Im übrigen waren sämmtliche Wochenbettsfunctionen vollständig normal. Trotzdem hatte sie abendliche Steigerungen bis 39,4 , bei morgendlichen Remissionen von 38,6 , die, nachdem die Nachwehen aufgehört hatten und der Uterus weicher geworden war, ohne dass sonstige Veränderungen im Befinden eintraten, nach und nach geringer wurden. Und so hat man bei genauer Beobachtung von Wöchnerinnen sehr häufig Gelegenheit, Temperatursteigerungen zu sehen, die mehr oder weniger uber die Norm hinausgehen und die man 
doch nicht gern als Fieber bezeichnet. Die Natur kennt eben keine scharfen Grenzen und von der normalen Temperatur zum Fieber gibt es zahllose Abstufungen, die natürlich nur in den seltensten Fällen mit dem Thermometer beobachtet werden.

Wird so durch die vermehrte örtliche Wärmeproduction auch die allgemeine Körpertemperatur etwas gesteigert, so muss, sobald die compensirenden Verhältnisse der Wärmeabgabe nach aussen eine Störung erleiden, sofort eine höhere Allgemeintemperatur entstehen. Dergleichen Störungen finden aber in den meisten pathologischen Fällen augenscheinlich statt, und mit ihnen ist die febrile Temperatursteigerung eingeleitet. Bei einem Gesunden wird die vermehrte Production durch vermehrte Ausgabe paralysirt, bei einem Kranken fällt das letztere weg, ja wird jedenfalls in vielen Fälen die Ausgabe verringert und somit ist un so mehr Grund zur Anbäufung der Wärme im Körper gegeben. Dass in pathologischen Fällen die Wärmeabgabe der Haut durch Verdunstung sowohl wie durch Wärmestrablung vermindert ist, erkennt die auf die trockne, kalte Haut eines Kranken mit beginnendem Schüttelfrost gelegte Hand leicht. Wodurch diese Contraction der Hautmuskeln, die die Gänsehaut bewirken, wodurch die Zusammenziehung der kleinsten Arterien, die durch die Blässe der Haut augenscheinlich wird, hervorgebracht wird, wollen wir hier nicht weiter erörtern und verweisen desswegen auf die Virchow'schen Auseinandersetzungen im Handbuch der speciellen Pathologie und Therapie, S. 32 u. f. Jedenfalls isł aber auch im stärksten Schüttelfrost die Verminderung der Wärme nur auf die äussersten Hautschichten beschränkt und ist schon im Beginn des Schuittelf́rostes dje Temperatur der Achselhöhle bedeutend erböbt *). Ich will, da von Anfang an genau beobachtete Schüttelfröste nicht so häufig sind, einen Fall, in dem ich ein sehr schnelles Steigen der Körpertemperatur durch zwei in die Achselhöhle und in die Vagina gelegte Thermometer genau beobachtete, anführen und bemerke nur, dass die Kranke eine Wöchnerin war, die am 24sten Tage des Wochenbetts mit einer Temperatur von 42,45 an einem pyämischen Milzabscess, der in die Pleurahöhle durchgebrochen war, starb. Die Thermometer blieben die ganze Zeit uber liegen und die Tem-

*) Liebermeister, a. a. 0. S. 14 . 
peratur wurde in Zeiträumen, die in der ersten Columne angegeben sind, notirt.

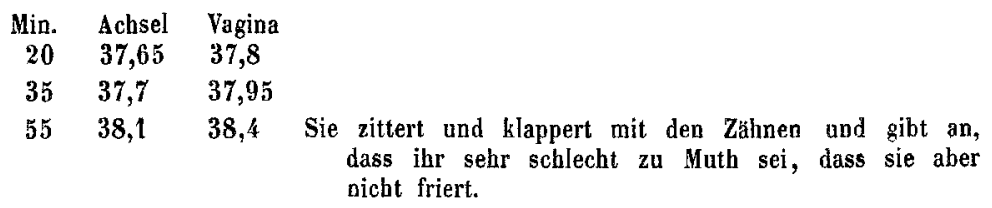

$6538,55 \quad 38,65$ Sehr starker Schüttelfrost. Sie klappert laut mit den Zảhnen. Haut trocken, an den Beinen deutliche Gänsehaut. Lippen blau, Gesicht, Füsse und Knie kalt. Sehr schlechtes subjectives Gefübl.

$75 \quad 39,3 \quad 39,3 \quad$ Vollständig ebenso.

$85 \quad 39,85 \quad 39,85$ Das suhjective Befinden ist weit besser. Zittern und Zähneklappern verschwunden. Sonst ebenso. Gänsehaut sehr deutlich.

$95 \quad 40,2 \quad 40,25$ Das subjective Befinden gut. Gänsebaut verschwunden. Lippen blau, Haut trocken, Nase, Füsse und Knien kalt. Kein Hitzegefühl.

$105 \quad 40,3 \quad 40,4 \quad$ Ebenso.

$115 \quad 40,35 \quad 40,4$ Stirn brennend heiss, Nase kalt, sonst ebenso.

$125 \quad 40,4 \quad 40,45$

135. 40,45 40,5 Knien und Nase warm, Füsse noch kalt.

$145 \quad 40,5 \quad 40,55$

$15540,55 \quad 40,6$ Nase heiss, Füsse noch kalt.

$165 \quad 40,65 \quad 40,65$

$175 \quad 40,7 \quad 40,7$ Füsse warm, die Haut ist trocken.

$185 \quad 40,8 \quad 40,75$

$19540,8 \quad 40,8$ Haut trocken.

$205 \quad 40,6 \quad 40,65$ Haut klebrig.

$215 \quad 40,6 \quad 40,67$

$22540,6 \quad 40,7$ Auf der Stirn kleine Schweisströpfchen.

$235 \quad 40,55 \quad 40,6$

$245 \quad 40,5 \quad 40,6$

$255 \quad 40,4 \quad 40,6$

$26540,35 \quad 40,45$ Die Haut ist klebrig, Schweisstropfen sind nur auf der Stirn.

Am anderen Tag hatte ich wieder Gelegenheit einen Schüttelfrost in seinen Anfängen zu beobachten, der hier, da ich zugleich Puls und Temperatur dabei beobachtete, gleichfalls noch seinen Platz finden mag.

$\begin{array}{rlccc}\text { Min. } & \text { Achsel } & \text { Vagina } & \text { Puls } & \text { Respir. } \\ \mathbf{2 0} & \mathbf{3 7 , 4} & \mathbf{3 7 , 6 5} & \mathbf{9 0} & \mathbf{2 9} \\ \mathbf{3 5} & \mathbf{3 7 , 4} & \mathbf{3 7 , 6 5} & & \\ \mathbf{5 5} & \mathbf{3 7 , 6 5} & \mathbf{3 7 , 9 5} & \mathbf{8 4} & \mathbf{2 0}\end{array}$


Min. Achsel Vagina Puls Respir.

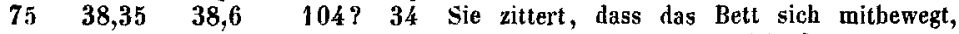
gibt aber an, dass sie nicht friert. Gänsehaut, Lippen blau, Stirn, Nase, Knien und Füsse kalt. Haut trocken. Der Puls ist so klein, dass er bei dem heftigen Zittern nicht genau zu zählen ist. Sie befindet sich subjectiv nicht schlecht.

$8538,95 \quad 38,95 \quad 100 \quad 34$ Zittert weniger, sonst ebenso. Puls sehr klein. Subjectives Befinden nicht schlecht.

$\begin{array}{lllll}90 & 39,2 & 39,2 & 98 & 32\end{array}$

$95 \quad 39,4 \quad 39,4 \quad 106 \quad 29$ Puls noch klein, sonst ebenso.

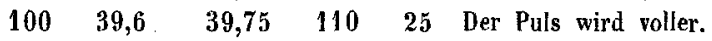

$110 \quad 39,75 \quad 39,85 \quad 134 \quad 34$

$\begin{array}{lllll}115 & 39,9 & 40,0 & 128 & 34\end{array}$

$125 \quad 40,05 \quad-\quad 118 \quad 33$

$135 \quad 40,2 \quad-\quad 118 \quad 23$

$145 \quad 40,4 \quad-\quad 12014$ Sehr tiefe Inspirationen. Die Haut ist trocken.

Die Thermometer werden entfernt und genau nach einer Stunde wieder eingelegt.

$\begin{array}{rlllll}20 & 41,4 & 41,5 & 132 & 41 & \begin{array}{c}\text { Kopfschmerzen, Befinden schlechter als vor- } \\ \text { her, Hitze, Durst, Haut trocken. }\end{array} \\ 40 & 41,2 & 41,25 & 138 & 40 & \text { Haut klebrig, Schweisstropfen auf der Stirn. } \\ 60 & 40,8 & 40,85 & 124 & 36 & \\ 80 & 40,45 & 40,55 & 126 & 38 & \\ 100 & 40,05 & 40,15 & 120 & 33 & \text { Schwitzt am ganzen Körper. } \\ 120 & 39,8 & 39,9 & 126 & 40 & \\ 140 & 39,75 & 39,85 & 120 & 36 & \text { Gesicht geröthet, sehr starker Schweiss. } \\ 160 & 39,5 & 39,55 & 119 & 28 & \\ 180 & 39,5 & 39,4 & 112 & 35 & \text { Schwitzt noch sehr stark. } \\ 200 & 39,15 & 39,2 & 112 & 34 & \text { Subjectives Befinden gut. } \\ 210 & 38,8 & 38,9 & 106 & 28 & \text { Noch starker Schweiss. } \\ 230 & 38,6 & 38,7 & 100 & 28 & \text { Etwas weniger Schweiss. } \\ 250 & 38,45 & 38,6 & 100 & 32 . & \end{array}$

Man sieht, dass die Temperatur der Achselhöhle anfangs etwas langsamer - wodurch die Liebermeister'schen oben citirten Deductionen auffallend bestätigt werden - dann aber in gleichem Schrill mit der Temperatur der Vagina steigt und dass sie dieselbe Höhe erreicht; und worauf es mir hier hauptsächlich ankommt, dass sofort mit dem Eintritt der Schweisssecretion die Temperatur zu fallen beginnt. Ich babe noch mehr Schüttelfröste mit dem Thermometer genau beobachtet, aber niemals, sobald die Haut anfing feucht zu werden, noch ein Steigen der Temperatur constatiren können. 
Wenn es somit auch einleuchtend ist, dass bei vermehrter Wärmeproduction und gleichbleibender oder gar verringerter Wärmeabgabe nach aussen eine Erböhung der allgemeinen Temperatur eintreten muss, so ist der Traube'sche Versuch, die febrile Temperatursteigerung einzig und allein durch Verhinderung der Wärmeabgabe $\mathrm{zu}$ erklären, als ein misslungener $\mathrm{zu}$ bezeichnen. Denn schon Immermann*), hat bevor Traube seine Theorie aufstellte, durch calorimetrische Messungen gezeigt, dass die im Fieber producirte und zur Steigerung der Körpertemperatur verwandte Wärmemenge bedeutender ist als die unter normalen Verhältnissen erzeugte, dass also die Verhinderung der Wärmeabgabe nicht ausreicht, die Temperaturerböbung zu erklären und Liebermeister bringt (a. a. 0.) neue auf calorimetrische Berechnungen basirte Beweise dafür bei. Immerhin aber wird es auf die Höhe des Fiebers von sehr bedeutendem Einfluss sein, ob die Wärmeabgabe nur wenig oder gar nicht vermehrt oder ob sie gar verringert ist.

Durch die dem Blut im Entzündungsheerd mitgetheilten Eigenschaften wird nun aher auch der Anstoss zur vermebrten Oxydation in allen übrigen Organen gegeben. Schon die höhere Wärme des kreisenden Blutes erhöbt alle Lebensvorgänge. „Es kann keinem Zweifel unterliegen, " sagt Weber a. a. 0 . S. 388 "dass bei einer höheren Temperatur alle organischen und namentlich formativen Prozesse lebhafter von Statten gehen. Die directe Beobachtung erweist, dass man durch Temperaturerhöhung die Bewegungen des Protoplasma der Zellen sehr erheblich zu beschleunigen und lebhafter zu machen im Stande ist." Wenn nun ferner auch "die Einleitung chemischer Verbindungen durch Wärme begünstigt wird," wenn es wahrscheinlich ist, dass die Affinitäten der Gewebe zu den Sauerstoffträgern des Blutes mit erhöhter Wärme sich steigern und die Oxydationsprozesse lebhafter vor sich geben, so ist es tinleuchtend, dass schon durch wärmeres Blut die Organe zu lebhafterem Stoffwechsel angeregt werden und dadurch selbst wieder vermehrte Wärme produciren.

Die Temperatursteigerung ist aber gewiss nicht die einzige Veränderung, die das Blut in entzündeten Organen erfäbrt. Für gewöhnlich reicht der mit den rothen Blutkörperchen den Geweben

*) De morbis febrilibus etc. Diss. inaug. Berol. 1860. S. 12 u. f. 
zugefithrte Sauerstoff aus, die verbrauchten nicht mehr functionsfähigen Stoffe nach und nach bis zu Harnstoff, Koblensäure und Wasser zu oxydiren und die dazwischenliegenden zahlreichen, leider noch so wenig gekannten Oxydationsstufen treten wohl immer nur ausnahmsweise als solche in das Blut uber *). Ist aber in einem Entzündungsheerd der Stoffverbrauch vermehrt, so kommt allerdings bei Beschleunigung des Kreislaufes zu derselben Zeit eine grössere Anzahl rother Blutkörperchen zu dem Gewebe und rielleicht verlieren dieselben auch in entzündeten Geweben ihren Sauerstoff vollständiger, so dass dieser mitunter zur Oxydation ausreichen mag - immerhin aber wird es eirie Grenze geben, bis zu der das zugeführte Blut die verbrauchten Stoffe zu oxydiren vermag, über die hinaus aber unvollständige Oxydationsstufen mit dem Blute fortgeführt werden. Welchen Einfluss diese in das Blut übergehenden Stoffe auf die Fiebererscheinungen haben, ist noch wenig aufgeklärt. Die schönen Experimente von 0 . Weber haben nachgewiesen, dass sowohl das Blut als die Parenchymsäfte eines Entziindungsheerdes selbst wieder entzündungserregende Ejgenschaften haben und es ist wahrscheinlich, dass es die in obiger Anmerkung genannten oder Stofe von derselben Bedentung sind, durch die diese Eigenschaft vermittelt wird.

Eine nicht unbedeutende Rolle wird aber jedenfalls auch der Verbrennungsprozess spielen, der mit dem Uebergang jener unvollständig verbrannten Stoffe in das Blut in dem Blute selbst weiter vor sich gehen muss. Das mit diesen Stoffen geschwängerte seines Sauerstoffgehaltes vollständig beraubte Blut kommt erst in den Lungen mit Sauerstoff wieder in Berührung. Ein grosser Theil derselben mag hier unter Mitwirkung der rothen Blutkörperchen verbrannt werden, ein gewisser Theil kann aber unter Umständen gewiss auch noch hier im Blut zurïckbleiben und wird dann dem arteriellen Blut einen Theil seines Sauerstoffs entziehen und es so weniger fähig machen, seine oxydirende Rolle vollständig zu spie-

*) Wenigstens kommen unter normalen Verbältnissen (s. Gorup-Besanez, Physiologische Chemie S. 290) im Blıt nur Spuren won flüchtigen Sänren an Alkalien gebunden vor, während unter pathologischen Verhältnissen Ameisensâure und Essigsäure, Harnsäure, Sarkin, Glutin, Leucin und Tyrosin, die alle als $\mathrm{Zwischenoxydationsstufen} \mathrm{der} \mathrm{regressiven} \mathrm{Metamorphose} \mathrm{zu} \mathrm{be-}$ trachten sind, im Blut gefunden werden. 
len. Ob die Wirkungen auf das Centrainervensystem, die wir in schweren fieberbaften Krankheiten beobachten, auf diese Weise durch Desoxydation des Blutes entstehen oder ob sie unmittelbare toxische Folgen jener unvollständig verbrannten Stoffe sind, mag dahingestellt bleiben; beides würde auch die schwersten Gehirnsymptome ungezwungen erklären können. Auch der Sauerstoffbunger heftig Fiebernder ist eine natürliche Folge des vermehrten Sauerstoffverbrauchs und die febrile Pulssteigerung ist, mag sie unmittelbar durch Einfluss auf den Vagus oder Sympathicus zu Stande kommen, mag sie nach Zimmermann eine Folge des vermebrten Sauerstoffhungers der Gewebe sein, leicht zu erklären. Dass der Tod auf diese Weise, lediglich durch die Höhe des Fiebers bedingt - wie er unzweifelhaft in einzelnen Krankheiten eintreten kann - in Folge vollständiger Ueberladung des Blutes mit oxydationsfähigen Substanzen, die allen Sauerstoff sofort für sich in Beschlag nehmen, die Scene schliessen kann, ist einleuchtend und findet eine Analogie in dem selten bei Menschen, häufiger bei gehetzten Thieren unter septicämischen Erscheinungen plötzlich eintretenden jedenfalls auch durch die massenhaften unvollständig verbrannten regressiven Stoffe bedingten Tode. Als Beweis gegen die Entstehung des Fiebers aus örtlichen Prozessen sind vielfach die acuten Exantheme und die pyämischen und septicämischen Erkrankungen angeführt worden. Dass aber die Pyämie stets örtliche Heerde hat, vou denen aus man das Fieber erklären kann, ist lange anerkannt. Will man aber auch die acuten Exantheme nicht, als Hautkrankheiten ansehen, so bleibt für diese und für die septicämischen Erkrankungen - von der bei letzteren constant auftretenden Enteritis abgesehen - immer noch das Blut als ursprüngIich erkranktes Gewebe übrig. Gewiss müssen, wenn hier gewöhnlich dem Blute die deletären Stoffe erst von einem Entzundungsheerd aus mitgetheilt werden, Fälle, in denen das Blut primär erkrankte, um so schneller und perniciöser verlaufen. Es steht nun nichts der Annahme entgegen, dass es Stoffe geben kann, die auf irgend einem Wege dem Blut zugeführt, die chemische Natur der rothen Blutkörperchen umzuändern im Stande sind, die einerseits sie fähig machen können, den Sauerstoff in vermehrtem Grade aufzunehmen und ihn lejchter abzugeben, dass es andererseits aber auch Stoffe geben kaan, die im Contact mit den Blutkörperchen die Lebens- 
fähigkeit derselben sofort vernichten. Während der erstere Fall die plötzlichen hohen Temperatursteigerungen erklären würde, könnte im zweiten. Fall natürlich keine Wärmesteigerung stattfinden, sondern müsste in intensiven Fällen der Tod sofort unter Temperaturabnahme erfolgen. Und in der That machen die Experimente von Weber und Billroth mit kohlensaurem Ammoniak und Buttersäure diese Ansicht sehr plausibel.

Die Abhängigkeit der Fiebertemperatur vom Entzündungsheerd wird ferner noch dadurch wahrscheinlich gemacht, dass die Höhe des Fiebers meistens mit der Extensität des ergriffenen Organes und der Intensität der Entzündung im Verhältniss steht. Ein Panaritium, eine entzündete Lymphdrüse machen nur geringe Fieberbewegungen, während wir im Tetanus, wo - wenn auch nur secundär - die gesammten willkürlichen Muskeln den Heerd für die Wärmeproduction bilden, die höchsten Temperaturgrade beobachten. Ja es scheint, als ob auch die Wichtigkeit des erkrankten Organs für die Blutbereitung und seine Beziebungen zum Kreislauf von wesentlichem Einfluss auf das Fieber sind. Wenigstens stehen das schnelle Steigen und die hohen Grade, die das Fieber z. B. bei Intermittens - wo die Milz jedenfalls primär oder secundär erkrankt ist - erreicht, in éigenthümlichem Gegensatz zu dem fast fieberlosen Verlauf einer Entzündung der Gehirnmasse.

Eine Lösung kann endlich die Fieberhitze nur finden, wenn wirklich das ursächlicbe Moment, die örtliche Wärmeproduction, die das veranlassende für die allgemeine Wärmeproduction war, wegfällt. Mit dem Nachlass, mit dem Ablauf der örtlichen Entzündung hört auch das Fieber auf. Diess kann, wenn eine typisch verlaufende Entzündung plötzlich aufbört, wenn zugleich mit dem Ablauf der Entzündung durch Scbweisse grössere Wärmeabgaben nach aussen auftreten, in Form der Krisis mit jähem Abfall erfolgen, oder es tritt, wenn die Entzündung sich nach und nach ermässigt, wenn mit dem Nachlassen derselben von Zeit zu Zeit kleine Steigerungen einhergehen, allmäblich in Form der Lysis auf.

Fassen wir zum Schluss die Hauptergebnisse unserer Betrachtungen zusammen, so haben wir gefunden: 
1) dass, wie die normale Oxydation der organischen Verbindungen die normale Körperwärme erzeugt, eine vermehrte oxydation derselben auch eine vermehrte Wärmemenge produciren muss.

2) dass demgemäss bei der activen Hyperämie, bei der der Stoffumsatz vermehrt und beschleunigt ist, eine Vermehrung der Wärmeproduction stattfinden muss.

3) dass diess in noch bedeutenderem Grade bei jeder frischen Entzündung der Fall ist.

4) dass jede örtliche Wärmesteigerung an und für sich auf die Körpertemperatur einen dieselbe erböhenden Einfluss ausübt.

5) dass dieser bei Hyperämien wohl immer, bei Entzündungen mitunter durch compensirende Einrichtungen zum grössten Theil aufgehoben wird.

6) dass geringere allgemeine Temperatursteigerungen auch wohl allein durch Mittheilung der örtlich producirten Wärmemenge von dem Gesammtorganismus hervorgebracht werden können.

7) dass aber bei der Entstehung höherer Fiebergrade woht stets sämmtliche Gewebe des Körpers, deren Stoffwechsel durch das abnorm veränderte Blut beschleunigt ist, mitbetheiligt sind.

Wenn diese theoretischen Schlüsse, die keineswegs den Anspruch machen, vollständig neu zu sein, durch eine Anzahl neuer Beobachtungen eine Stütze gefunden und diese Zeilen zur Sicherstellung der Lehre von der Entzuindung und vom Fieber einen neuen Baustein beigetragen haben, so ist ihr Zweck damit erreicht. 